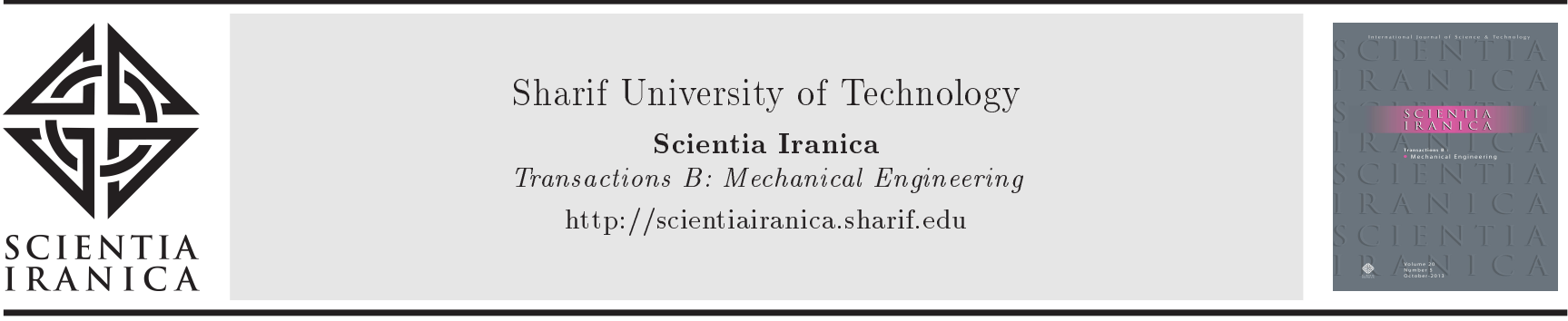

\title{
Hybrid integral transform and $p$-version FEM for thermo-mechanical analysis of a functionally graded piezoelectric hollow cylinder subjected to asymmetric loads
}

\author{
M. Dehghan, A. Moosaie*, and M.Z. Nejad \\ Department of Mechanical Engineering, Yasouj University, Yasouj, P.O. Box 75914-353, Iran.
}

Received 20 July 2020; received in revised form 4 November 2020; accepted 11 January 2021

\author{
KEYWORDS \\ FFT; \\ Higher-order finite \\ elements; \\ Piezoelectric cylinder; \\ Asymmetric thermal \\ loads.
}

\begin{abstract}
The value of this ingenious study is to be a pioneer in providing a combination of Fast Fourier Transform (FFT) and $p$-version of finite element method for the electrothermo-elastic analysis of a thick hollow cylinder under asymmetric thermal loadings. In shells of revolution, the proposed FFT- $p$ FE method is accompanied by a significant decrease in computational costs. Due to the problem periodicity of these types of structures, the FFT technique is used to discretize the governing equations into a set of harmonics. Each harmonic is then partitioned using higher-order finite elements. Hierarchical finite elements based on Legendre polynomial interpolation functions are utilized to discretize 2D governing equations of a Functionally Graded Piezoelectric (FGP) cylinder. 3D governing equations of an FGP hollow cylinder are then discretized by using the higher-order Lagrangian finite elements. The effects of FFT grid-size and the order of the interpolation functions on the convergence behavior of the proposed mixed FFT- $p$ FE method are investigated. The material properties, except the Poisson's ratio, are considered to vary along the radius of the cylinder. The governing equations are derived using the principle of virtual displacements. For a 3D FGP hollow cylinder, the influence of axially and circumferentially non-symmetric thermal loadings is presented in contour plots.
\end{abstract}

(C) 2021 Sharif University of Technology. All rights reserved.

\section{Introduction}

Over the recent years, modern industrial productions are concerned with the layered piezoelectric structures produced in the form of a thick cylinder. They can be used as smart devices, e.g. in engineering applications with active control. In this class of piezoelectric struc-

\footnotetext{
*. Corresponding author. Tel.: +98 7433221711 ; Fax: +98 743 322 1711;

E-mail address: moosaie@yu.ac.ir (A. Moosaie)
}

tures, material properties suddenly change passing through the cylinder thickness from a layer to the other. So, the interface between two dissimilar materials is often accompanied by stress concentration and huge thermal stresses [1]. Consequently, an advanced class of heterogeneous composite materials has been developed to overcome these issues in which the mechanical properties continuously and gradually vary from one point to the other [1-4]. Therefore, the electro-thermo-elastic analysis of the Functionally Graded Piezoelectric Materials (FGPMs) seems to be attractive. Indeed, there are several numerical and analytical studies concerned with these problems, as briefly reviewed in the sequel. 
Ootao et al. [5] has presented an analytical approach for transient thermo-electro-elastic analysis of Functionally Graded Piezoelectric (FGP) hollow cylinder. A three-dimensional exact solution of FGP shells under cylindrical bending has been performed by $\mathrm{Wu}$ and Syu [6] using the perturbation method. The effect of thermo-electro-mechanical loading on a Functionally Graded (FG) shell in the form of a thick sphere bonded with piezoelectric layers is investigated by Alashti et al. [7]. Due to their ability in withstanding high-pressure loadings with respect to their weight, shells of revolution have received more attention in numerous applications of structural engineering. Recently, the authors have derived a set of field equations for a FGP shell of revolution with arbitrary curvature and variable thickness using tensor analysis in curvilinear coordinate systems [8]. Nejad et al. [9] have developed a general formulation for thermo-elastic analysis of a FG thick shell of revolution with arbitrary curvature and variable thickness by using higher-order shear deformation theory. A semianalytical method has been frequently used by Santos and his co-workers [10-14] for the three-dimensional analysis of axisymmetric shells of revolution. These articles especially emphasized the coupling between the symmetric and anti-symmetric terms in the truncated Fourier expansion of the dependent variables and the loading. It was also demonstrated that the material properties have a significant effect when comparing coupled and uncoupled results. The analysis of shells of revolution is often accompanied by a Fourier representation of unknown variables and the boundary conditions along the circumferential direction. For example, Noor and Peters [15] used this ability to perform bending and free vibration analysis of layered anisotropic shells of revolution. The coupling effect between symmetric and anti-symmetric modes for composite laminated shells of revolution was investigated by using the aforementioned semi-analytical method [16]. Sivadas and Ganesan [17] used double Fourier series approximation and Finite Element Method (FEM) to study the coupling effect of symmetric and asymmetric vibration modes of laminated composite shells of revolution. Fourier series is used by Loghman et al. [18] for thermo-elastic analysis of a FG cylinder under asymmetric thermal and mechanical loadings subjected to a uniform magnetic field. In the 3D investigation of the FGP cylindrical shells, Chen et al. [19] analyzed the dynamic response of an FGP hollow cylinder that encompasses a compressible fluid. Liang et al. [20] introduced a semi-analytical method using Laplace transform and the Differential Quadrature (DQ) method to study the dynamic behavior of FGP cylindrical panels under different boundary conditions. Propagating the waves in reinforced FG porous plates was investigated by Gao et al. [21] using Classical Plate
Theory (CPT), First-order Shear Deformation Theory (FSDT), and Higher-order Shear Deformation Theory (HSDT).

Each numerical method may be inherently accompanied by some deficiencies regarding the modeling of geometry, the discretization, and the satisfaction of boundary conditions. Semi-analytical and mixed methods are considered to be appropriate to overcome these shortcomings. In this regard, the Fourier spectral method can provide an appropriate base through defining the trial and test functions [22]. Dehghan and Baradaran [23] and Malekzadeh and Karami [24] combined the FEM and DQ technique to gain the ability of mixed method in modeling the complex geometry and boundary conditions as well as the fast convergence. The time-dependency in a nonlinear thermal problem was removed by an effective combination of the Laplace transform and FEM by Lin and Chen [25]. Recently, the Laplace transform technique and multi-scale FEM were combined to solve coupled partial differential equations of the flow in a dualpermeability system by Liu et al. [26]. Entezari et al. [27] used the capability of Carrera Unified Formulation to thermo-elastic wave propagation analysis of FG disks. Through this method, one-dimensional finite elements are used to interpolate the displacement and temperature field variables to discretize the control equations. A layerwise differential quadrature method (LW-DQM) was combined with a Non-Uniform Rational B-Spline (NURBS) multi-step time integration scheme by Heydarpour et al. [28]. They investigated thermal shock wave effects in FG panels bonded with piezoelectric layers. An ingenious effort, using the Rayleigh-Ritz method, has been made by Qin et al. [29-31] for analyzing the plates and shells reinforced by Carbon Nano-Tubes (CNT) and graphene platelets (GPLs). Nowadays, the application of porous materials and nanocomposites (CNT-filled polyethylene) is increasing, especially in the offshore industries. So far, some measures have been taken to investigate these materials $[32,33]$. A vast review of the literature reveals that the electro-thermo-elastic analysis of FGP shells of the revolution by mixed numerical FFT- $p$ FE method is scarce. So far, the transient thermo-elastic analysis of the disk brake has been studied by Fast Fourier Transform (FFT) and FEM [34-36]. Recently, Jin et al. [37] have developed a modified Fourier series solution for vibration analysis of the shells of revolution. Mohazzab and Dozio [38,39] employed the spectral collocation method for the prediction of natural frequencies of laminated curved panels and skew plates, respectively. In this regard, Xie et al. [40] employed the spectral collocation method for free vibration analysis of the composite shell supported by the elastic foundation.

Here, the FFT and the $p$-version of the FEM are 
combined to gain more advantages in the analysis of shells of revolution. The size and shape of the elements and the approximation properties of the solution space have significant effects on the quality of the finite element solution. The desirable precision in the finite element procedure is obtained by using $h$ - and $p$ version techniques. In the $p$-version technique, this can be achieved by increasing the order of shape functions. Shape functions for a one-dimensional hierarchic element are produced by integrating Legendre polynomials [41]. A major property of these shape functions is favorable orthogonality that leads to sparse and well-conditioned stiffness matrices [42]. Yu et al. [43] used hierarchical finite beam elements for static and dynamic analysis. The rate of convergence in the Timoshenko beam was improved by Tai and Chan [44] by considering Legendre-based hierarchic shape functions. Green's-function-based finite element formulation (HSF-FEM) is utilized by Wang and Qin [45] for simulating bioheat transfer in the human eye and by Cao et al. [46] for analyzing three-dimensional elastic problems with body forces.

The FFT- $p$ FE method proposed in this paper can be appropriately recognized as a semi-analytical technique since it attains higher precision and has less computational cost in comparison with $3 \mathrm{D}$ traditional $\mathrm{FE}$ computer programs. In fact, by simplifying the threedimensional governing equation to the two-dimensional governing equation, the calculation workload can be reduced. In addition, the proposed mixed method benefits from the advantages of the FFT algorithm. The discrete transform can be computed using matrixvector multiplication with the $2 N^{2}$ operations. The FFT technique which is successfully implemented in this paper has an operation count with a leading term $(5 / 2) N \log _{2} N$. From a numerical point of view, it is accompanied by considerable computational time savings. The computational routines for FFT and iFFT are available in the software package called 'FFTW3'. It is a ' $C$ ' subroutine library for computing the discrete Fourier transform in one or more dimensions. In general, restrictions of the present mixed-method can be explained from two points of view:

1. The grid size of the FFT method should be uniform along the circumferential direction;

2. Periodicity of the geometry (which is automatically satisfied for the periodic domains, such as the cylinder).

In this paper, the following steps are considered. First, the 2D heat conduction and electro-thermo-elastic Partial Differential Governing Equations (PDGEs) of the FGP hollow cylinder with plane strain assumption are discretized using the proposed FFT- $p$ FE method. The discrete Fourier Transform technique is used to project the PDGEs from real space into the Fourier space and vice versa. Hierarchical finite elements are introduced here for discretizing the meridian section of the cylinder. In the next step, we deal with 3D thermo-electro-elastic analysis of an FGP hollow cylinder. The meridian section of the cylinder is divided into subdomains called the second-order 2D finite elements of the Lagrange family. According to the existing algorithm, numerical results related to the temperature field are imported as input data for the electro-thermoelastic analysis of the FGP shell. Finally, the inverse Fast Fourier Transform (FFT) technique is used to invert the obtained results into real space.

\section{Problem formulation in $2 \mathrm{D}$ space using hierarchic elements}

\subsection{The heat conduction problem}

In a thermo-elastic analysis, the primary step is to extract the distribution of temperature field within the physical domain of the problem, to which is imposed appropriate boundary conditions. Therefore, the steady-state heat conduction equation is presented here for an FGP hollow cylinder in the absence of heat generation. This equation can be readily derived from the energy conservation law along with the Fourier heat conduction law. For an FG cylinder, this equation in radial and circumferential directions reads [8].

$$
\begin{gathered}
\lambda_{r} \frac{\partial^{2} T}{\partial r^{2}}+\frac{1}{r} \lambda_{r} \frac{\partial T}{\partial r}+\lambda_{r} \frac{1}{r^{2}} \frac{\partial^{2} T}{\partial \theta^{2}}+\frac{d \lambda_{r}}{d r} \frac{\partial T}{\partial r}=0, \\
a \leq r \leq b, \quad 0<\theta \leq 2 \pi .
\end{gathered}
$$

In this work, the following Dirichlet boundary conditions are assumed:

$$
\begin{aligned}
& T(r=a, \theta)=T_{i}(\theta), \\
& T(r=b, \theta)=T_{o}(\theta),
\end{aligned}
$$

in which $T$ is the temperature field, and $\lambda_{r}=\lambda_{r}(r)$ is the radially-variable thermal conductivity coefficient. $T_{i}$ and $T_{o}$ are temperature distributions at inner and outer surfaces of the cylinder whereas $a$ and $b$ indicate inner and outer radii of the cylinder, respectively. Here, the thermal conductivity coefficient, $\lambda_{r}(r)$, is considered to be graded according to a power function of the form

$$
\lambda_{r}=\lambda_{r 0} \bar{r}^{m}, \quad \bar{r}=\frac{r}{r_{b}},
$$

where $\lambda_{r 0}$ is some material constant regarding thermal conductivity. $m$ is the power-law index of the material. As mentioned earlier, it can be considered that the temperature field is periodic in the circumferential 


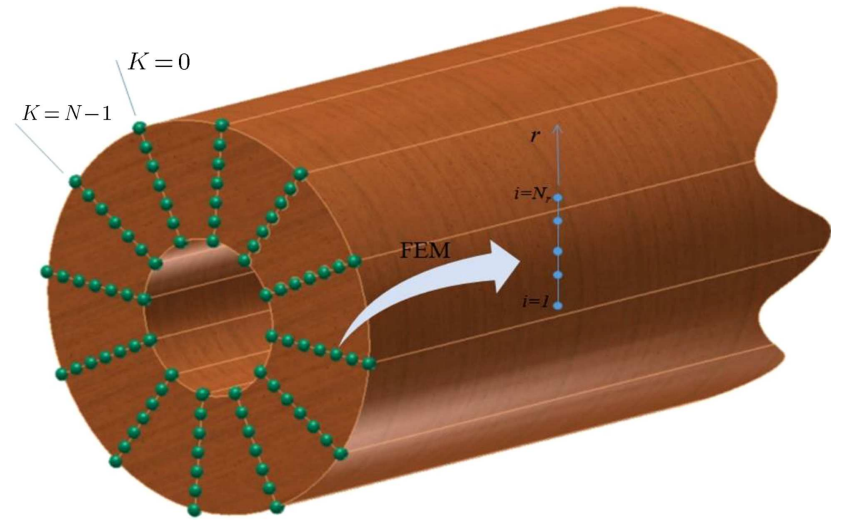

Figure 1. Discretized geometry of an infinitely long Functionally Graded Piezoelectric (FGP) cylinder in the presence of finite element grids and Fast Fourier Transform (FFT) harmonics.

direction, so periodic boundary condition is applied in this direction. At first, Fourier transform is used for solving this partial differential equation. For this purpose, the temperature field in Eq. (1) is replaced by the approximation function, including discrete Fourier coefficients. As shown in Figure 1, the temperature field is defined at discrete points $\theta_{k}$ with $k=0,2, \cdots, N-$ 1. In Fourier space, the integer parameter $k$ is the wavenumber in the circumferential direction. The selected approximation function is defined as:

$$
\begin{aligned}
& T\left(r, \theta_{j}\right)=T_{j} \approx \sum_{k=-\frac{N}{2}}^{\frac{N}{2}} \hat{T}^{k}(r) e^{i k \theta_{j}} . \\
& j=0, \ldots, N-1 .
\end{aligned}
$$

By substituting this ansatz into Eq. (1), the heat equation and its related boundary conditions in radial direction become:

$$
\begin{aligned}
& \lambda_{r} \frac{d^{2} \hat{T}^{k}}{d r^{2}}+\frac{\lambda_{r}}{r} \frac{d \hat{T}^{k}}{d r}+\lambda_{r}{ }^{\prime} \frac{d \hat{T}^{k}}{d r}-\lambda_{r} \frac{k^{2}}{r^{2}} \hat{T}^{k}=0, \\
& \quad \text { for } \quad k=0, \ldots, N-1, \\
& \hat{T}^{k}\left(r=a, \theta_{k}\right)=\hat{T}_{i}\left(\theta_{k}\right), \quad \hat{T}^{k}\left(r=b, \theta_{k}\right)=\hat{T}_{o}\left(\theta_{k}\right),
\end{aligned}
$$

where $(\ldots)^{\prime}$ denotes the first derivative of any arbitrary function with respect to the variable $r$.

The method of weighted residuals provides a framework for solving partial differential equations approximately. The general form of the partial differential equation to be solved is typically written as:

$$
\tilde{\Gamma}\left(\hat{T}^{k}(r)\right)-f=R
$$

In general, an approximate solution, say $\hat{T}^{k}$, does not exactly satisfy Eq. (5). Thus, the weighted residual method is utilized to find a solution such that the residual $R$ is minimized in the sense of a weighted integral:

$$
\int_{\Omega^{e}} \boldsymbol{w} R d \Gamma=0,
$$

where, $\boldsymbol{w}$ is a vector of weighting functions and,

$$
d \Gamma=r d r
$$

Substituting Eq. (7) into Eq. (8) yields:

$$
\begin{aligned}
& \int_{\Omega^{e}} w_{q}\left[\frac{1}{r} \frac{d}{d r}\left(r \frac{d \hat{T}^{k}}{d r}\right)+\frac{\lambda_{r}{ }^{\prime}}{\lambda_{r}} \frac{d \hat{T}^{k}}{d r}-\frac{k^{2}}{r^{2}} \hat{T}^{k}\right] r d r=0 \\
& \text { for } k=0, \ldots, N-1, \quad q=1,2, \ldots, N_{r}
\end{aligned}
$$

By assuming $w_{q}$ to be sufficiently differentiable weight functions, the original equation can be easily transformed into the weak form using the integration by parts technique. In the finite element context and based on the Bubnov-Galerkin method, the weight functions $w_{i}$ are the same as the interpolant polynomials. In each hierarchical element, it is presumed that the temperature variable $\hat{T}^{k}(r)$ is approximated as a series function containing the product of basis functions $\phi_{j}^{e}$ and nodal values:

$$
\hat{T}^{k}(r)=\sum_{j=1}^{N_{r}} \psi_{j}(r) \hat{T}_{j}^{k},
$$

where $\hat{T}_{j}^{k}$ are the nodal values regarding the temperature field in the complex domain. Higher-order Legendre polynomials are considered as the basis functions $\psi_{j}$ in a hierarchical element. More details of these basis functions and hierarchical elements will be introduced in the next section. Now, the stiffness matrix can be obtained using the Gauss-Legendre quadrature technique and is arranged in matrix form as:

$$
[\hat{K}]\left\{\hat{T}^{k}\right\}=\{\hat{Q}\}
$$

where $[\hat{K}]$ and $\{\hat{Q}\}$ are stiffness matrix and temperature gradient vector which can be written as follows:

$$
\begin{aligned}
& \hat{K}_{i j}=\int_{\Omega_{e}}\left(r \frac{d \psi_{i}^{e}}{d r} \frac{d \psi_{j}^{e}}{d r}+\frac{k^{2}}{r} \psi_{i}^{e} \psi_{j}^{e}-r \frac{\lambda_{r}{ }^{\prime}}{\lambda_{r}} \psi_{i}^{e} \frac{d \psi_{j}^{e}}{d r}\right) d r, \\
& \hat{Q}_{i}=\oint_{\Gamma^{e}} \psi_{i}^{e} q_{n} d s \\
& q_{n}=\left(\lambda_{r} \frac{d \hat{T}_{k}}{d r} n_{r}\right) .
\end{aligned}
$$

Solving this set of equations yields the nodal values of the temperature field for each wavenumber. 


\subsection{The electro-thermo-elastic problem 2.2.1. FFT-pFE analysis}

In this section, the mixed FFT- $p$ FE method is used to perform electro-thermoelastic analysis of a hollow FGP cylinder. For this purpose, a hollow cylinder, which its stress is negligible at the reference temperature, operates in a thermal environment. Thermal loading together with mechanical and electrical constraints causes thermal stresses in the shell. PDGEs of the FGP hollow cylinder and related boundary conditions are derived using Hamilton's principle. It should be noted that the Fourier spectral method acts upon strong form and the FEM needs to be executed on the weak form of the PDGEs. So, Hamilton's principle can appropriately provide these conditions, simultaneously. A special case of Hamilton's principle that deals with elastic mediums is known as the principle of minimum total potential energy. For an FGP cylinder, it takes the following form [8]:

$$
\delta \Pi=\delta(V+H)=0,
$$

where $\delta$ is the variation symbol and $\Pi$ is called the total potential energy of the elastic body. The electric enthalpy $H$ and the energy of the applied loads $V$ can be expressed as:

$$
\begin{aligned}
H= & \frac{1}{2} \iiint_{v}\left[\{\sigma\}^{T}\{\varepsilon\}-\{D\}^{T}\{E\}\right] \mathrm{d} v, \\
V= & -\iint_{\Gamma} u_{r}\left\{r \sigma_{r r} n_{r}+\sigma_{r \theta} n_{\theta}\right\} d s \\
& -\iint_{\Gamma} u_{\theta}\left\{r \sigma_{r \theta} n_{r}+\sigma_{\theta \theta} n_{\theta}\right\} d s .
\end{aligned}
$$

The kinematic relationships between strain components and displacement field are defined as:

$$
\begin{aligned}
& \varepsilon_{r r}=\frac{\partial u_{r}}{\partial r}, \quad \varepsilon_{\theta \theta}=\frac{1}{r}\left(u_{r}+\frac{\partial u_{\theta}}{\partial \theta}\right), \\
& \varepsilon_{r \theta}=\frac{1}{2}\left(\frac{1}{r} \frac{\partial u_{r}}{\partial \theta}+\frac{\partial u_{\theta}}{\partial r}-\frac{u_{\theta}}{r}\right), \\
& \varepsilon_{z z}=\varepsilon_{r z}=\varepsilon_{\theta z}=0, \quad\left\{\begin{array}{l}
E_{r}=-\frac{\partial \varphi}{\partial r} \\
E_{\theta}=-\frac{1}{r} \frac{\partial \varphi}{\partial \theta} \\
E_{z}=0
\end{array}\right.
\end{aligned}
$$

where $\varepsilon_{i j}(i, j=r, \theta, z)$ are the strain components in the cylindrical coordinate system. $u_{r}$ and $u_{\theta}$ are displacement components in radial and circumferential directions, respectively. Moreover, $E_{i}(i=r, \theta, z)$ represents the electric field corresponding to the electric potential $\varphi$. The plane-strain electro-thermo-elastic constitutive relations for an isotropic FGP hollow cylinder can be written as:

$$
\begin{aligned}
\left\{\begin{array}{l}
\sigma_{r r} \\
\sigma_{\theta \theta} \\
\sigma_{z z} \\
\sigma_{r \theta}
\end{array}\right\}= & {\left[\begin{array}{cccc}
C_{11} & C_{12} & C_{13} & 0 \\
C_{21} & C_{22} & C_{23} & 0 \\
C_{31} & C_{32} & C_{33} & 0 \\
0 & 0 & 0 & C_{44}
\end{array}\right]\left\{\begin{array}{c}
\varepsilon_{r r}-\alpha_{r} T \\
\varepsilon_{\theta \theta}-\alpha_{\theta} T \\
-\alpha_{z} T \\
2 \varepsilon_{r \theta}
\end{array}\right\} } \\
& -\left[\begin{array}{cc}
e_{11} & 0 \\
e_{21} & 0 \\
e_{31} & 0 \\
0 & e_{42}
\end{array}\right]\left\{\begin{array}{l}
E_{r} \\
E_{\theta}
\end{array}\right\},
\end{aligned}
$$

$$
\begin{aligned}
\left\{\begin{array}{l}
D_{r} \\
D_{\theta}
\end{array}\right\}= & {\left[\begin{array}{ccc}
e_{11} & e_{21} & 0 \\
0 & 0 & e_{42}
\end{array}\right]\left\{\begin{array}{l}
\varepsilon_{r r} \\
\varepsilon_{\theta \theta} \\
2 \varepsilon_{r \theta}
\end{array}\right\} } \\
& +\left[\begin{array}{cc}
\eta_{11} & 0 \\
0 & \eta_{22}
\end{array}\right]\left\{\begin{array}{l}
E_{r} \\
E_{\theta}
\end{array}\right\}+\left\{\begin{array}{l}
\mathrm{P}_{1} \\
\mathrm{P}_{2}
\end{array}\right\} T,
\end{aligned}
$$

where $\sigma_{i j}(i, j=r, \theta, z)$ and $D_{i}(i=r, \theta)$ represent stress components and electric displacements. $\alpha$ is the coefficient of thermal expansion. $e_{i j}, \eta_{i j}$ and $\mathrm{P}_{i}$ denote the piezoelectric, dielectric and pyroelectric constants, respectively. In this work, the material properties are considered to be graded along the $r$-direction as:

$$
\left\{\begin{array}{l}
C_{i j}=C_{i j}^{0} \bar{r}^{l}, \quad e_{i j}=e_{i j}^{0} \bar{r}^{l}, \quad \eta_{i j}=\eta_{i j}^{0} \bar{r}^{l} \\
\alpha_{i}=\alpha_{i}^{0} \bar{r}^{b}, \quad \mathrm{P}_{i}=\mathrm{P}_{i}^{0} \bar{r}^{l+b},
\end{array}\right.
$$

where $l$ is a power-law index of the elastic constants, the piezoelectric coefficient, and the dielectric constants. $b$ indicates a power-law index for the coefficient of thermal expansion. Both $l$ and $b$ inhomogeneous parameters are considered to be applied in the pyroelectric constants. Substituting Eqs. (13)-(15) into Eq. (12) yields the following integral form:

$$
\begin{aligned}
\iint\{ & {\left[C_{11}\left(\frac{\partial u_{r}}{\partial r}\right)+C_{12}\left(\frac{u_{r}}{r}+\frac{1}{r} \frac{\partial u_{\theta}}{\partial \theta}\right)-e_{11}\left(-\frac{\partial \varphi}{\partial r}\right)\right.} \\
& \left.-\beta_{1} T\right] \frac{\partial w_{1}}{\partial r}+\left[C_{12}\left(\frac{\partial u_{r}}{\partial r}\right)+C_{22}\left(\frac{u_{r}}{r}+\frac{1}{r} \frac{\partial u_{\theta}}{\partial \theta}\right)\right. \\
& \left.-e_{21}\left(-\frac{\partial \varphi}{\partial r}\right)-\beta_{2} T\right]\left(\frac{w_{1}}{r}+\frac{1}{r} \frac{\partial w_{2}}{\partial \theta}\right) \\
& +\left[C_{44}\left(\frac{1}{r} \frac{\partial u_{r}}{\partial \theta}+\frac{\partial u_{\theta}}{\partial r}-\frac{u_{\theta}}{r}\right)-e_{42}\left(-\frac{1}{r} \frac{\partial \varphi}{\partial \theta}\right)\right] \\
& +\left(\frac{1}{r} \frac{\partial w_{1}}{\partial \theta}+\frac{\partial w_{2}}{\partial r}-\frac{w_{2}}{r}\right)-\left[e_{11}\left(\frac{\partial u_{r}}{\partial r}\right)\right. \\
& \left.+e_{21}\left(\frac{u_{r}}{r}+\frac{1}{r} \frac{\partial u_{\theta}}{\partial \theta}\right)+\eta_{11}\left(-\frac{\partial \varphi}{\partial r}\right)+\mathrm{P}_{1} T\right] \\
& \left(-\frac{\partial w_{3}}{\partial r}\right)-\left[e_{42}\left(\frac{1}{r} \frac{\partial u_{r}}{\partial \theta}+\frac{\partial u_{\theta}}{\partial r}-\frac{u_{\theta}}{r}\right)\right. \\
+ & \left.\left.\eta_{22}\left(-\frac{1}{r} \frac{\partial \varphi}{\partial \theta}\right)+\mathrm{P}_{2} T\right]\left(-\frac{\partial w_{3}}{r}\right)\right\} r d r d \theta=0
\end{aligned}
$$


where:

$$
\begin{gathered}
w_{1}=\delta u_{r}, w_{2}=\delta u_{\theta}, w_{3}=\delta \varphi \\
\left\{\begin{array}{l}
\beta_{1}=C_{11} \alpha_{r}+C_{12} \alpha_{\theta}+C_{13} \alpha_{z} \\
\beta_{2}=C_{12} \alpha_{r}+C_{22} \alpha_{\theta}+C_{23} \alpha_{z} .
\end{array}\right.
\end{gathered}
$$

Now, the Fourier transform method can be used to discretize governing equations of an FGP cylinder into a set of harmonics in the circumferential direction. For this purpose, according to the FFT method, the approximation functions are considered for displacement components and the electric potential of the cylinder:

$$
\left\{\begin{array}{l}
u_{r}(r, \theta)=\sum_{k=1}^{N} \sum_{j=1}^{N_{r}} \psi_{j}(r)\left(\hat{u}_{r}\right)_{j}^{k} e^{i k \theta}, \\
u_{\theta}(r, \theta)=\sum_{k=1}^{N} \sum_{j=1}^{N_{r}} \psi_{j}(r)\left(\hat{u}_{\theta}\right)_{j}^{k} e^{i k \theta} \\
\varphi(r, \theta)=\sum_{k=1}^{N} \sum_{j=1}^{N_{r}} \psi_{j}(r)(\hat{\varphi})_{j}^{k} e^{i k \theta}
\end{array}\right.
$$

where $\left(\hat{u}_{r}\right)_{j}^{k}, \quad\left(\hat{u}_{\theta}\right)_{j}^{k}$ and $(\hat{\varphi})_{j}^{k}$ are nodal values of displacement components and electric potential in the Fourier space. Using the integration by parts technique with respect to the circumferential variable $\theta$, we can transform a part of the governing equations into a strong form. By substituting approximation functions of Eq. (19) into Eq. (17), for each harmonic $k$ with $k=1, \cdots, N$, we have:

$$
\begin{aligned}
& \left(\delta \hat{u}_{r}\right)_{i}^{k}: C_{11} \sum_{j=1}^{N_{r}} D_{i j}\left(\hat{u}_{r}\right)_{j}^{k} \\
& +C_{12}\left(\sum_{j=1}^{N_{r}} A_{i j}\left(\hat{u}_{r}\right)_{j}^{k}+\sum_{j=1}^{N_{r}} G_{i j}\left(\left(\hat{u}_{r}\right)_{j}^{k}+i k\left(\hat{u}_{\theta}\right)_{j}^{k}\right)\right) \\
& +C_{22} \sum_{j=1}^{N_{r}} B_{i j}\left(\left(\hat{u}_{r}\right)_{j}^{k}+i k\left(\hat{u}_{\theta}\right)_{j}^{k}\right) \\
& +C_{44}\left(\sum_{j=1}^{N_{r}} B_{i j}\left(k^{2}\left(\hat{u}_{r}\right)_{j}^{k}+i k\left(\hat{u}_{\theta}\right)_{j}^{k}\right)\right. \\
& \left.-i k \sum_{j=1}^{N_{r}} A_{i j}\left(\hat{u}_{\theta}\right)_{j}^{k}\right)^{k}-e_{42} k^{2} \sum_{j=1}^{N_{r}} B_{i j} \hat{\varphi}_{j}^{k} \\
& \left(\delta \hat{u}_{\theta}\right)_{i}^{k}:-i k C_{12} \sum_{j=1}^{N_{r}} A_{i j}\left(\hat{u}_{r}\right)_{j}^{k}-C_{22} \sum_{j=1}^{N_{r}} B_{i j} \\
& \left(i k\left(\hat{u}_{r}\right)_{j}^{k}-k^{2}\left(\hat{u}_{\theta}\right)_{j}^{k}\right)-C_{44}\left(\sum_{j=1}^{N_{r}} B_{i j}\right.
\end{aligned}
$$

$$
\begin{aligned}
& \left(i k\left(\hat{u}_{r}\right)_{j}^{k}-\left(\hat{u}_{\theta}\right)_{j}^{k}\right)+\sum_{j=1}^{N_{r}} A_{i j}\left(\hat{u}_{\theta}\right)_{j}^{k}-\sum_{j=1}^{N_{r}} \\
& \left.G_{i j}\left(i k\left(\hat{u}_{r}\right)_{j}^{k}-\left(\hat{u}_{\theta}\right)_{j}^{k}\right)-\sum_{j=1}^{N_{r}} D_{i j}\left(\hat{u}_{\theta}\right)_{j}^{k}\right) \\
& -i k e_{21} \sum_{j=1}^{N_{r}} A_{i j} \hat{\varphi}_{j}^{k}-i k e_{42} \sum_{j=1}^{N_{r}}\left(B_{i j}-G_{i j}\right) \hat{\varphi}_{j}^{k} \\
& +i k \beta_{2} \sum_{j=1}^{N_{r}} E_{i j} \hat{T}_{j}^{k}=0,
\end{aligned}
$$

$\delta \hat{\varphi}_{i}^{k}:$

$$
\begin{aligned}
& e_{11} \sum_{j=1}^{N_{r}} D_{i j}\left(\hat{u}_{r}\right)_{j}^{k}+e_{21} \sum_{j=1}^{N_{r}} G_{i j}\left(\left(\hat{u}_{r}\right)_{j}^{k}+i k\left(\hat{u}_{\theta}\right)_{j}^{k}\right) \\
& -\eta_{11} \sum_{j=1}^{N_{r}} D_{i j} \hat{\varphi}_{j}^{k}-\eta_{22} k^{2} \sum_{j=1}^{N_{r}} B_{i j} \hat{\varphi}_{j}^{k}+e_{42} \\
& \left(k^{2} \sum_{j=1}^{N_{r}} B_{i j}\left(\hat{u}_{r}\right)_{j}^{k}+i k \sum_{j=1}^{N_{r}} B_{i j}\left(\hat{u}_{\theta}\right)_{j}^{k}-i k \sum_{j=1}^{N_{r}} A_{i j}\left(\hat{u}_{r}\right)_{j}^{k}\right) \\
& +\mathrm{P}_{1} \sum_{j=1}^{N_{r}} F_{i j} \hat{T}_{j}^{k}-i k \mathrm{P}_{2} \sum_{j=1}^{N_{r}} E_{i j} \hat{T}_{j}^{k}=0,
\end{aligned}
$$

where:

$$
\begin{aligned}
A_{i j} & =\int \psi_{i}\left(\frac{d \psi_{j}}{d r}\right) d r, \quad B_{i j}=\int\left(\frac{1}{r}\right) \psi_{i} \psi_{j} d r \\
D_{i j} & =\int\left(\frac{d \psi_{i}}{d r}\right)\left(\frac{d \psi_{j}}{d r}\right) r d r, \quad E_{i j}=\int \psi_{i} \psi_{j} d r \\
F_{i j} & =\int\left(\frac{d \psi_{i}}{d r}\right) \psi_{j} r d r, \quad G_{i j}=\int\left(\frac{d \psi_{i}}{d r}\right) \psi_{j} d r .
\end{aligned}
$$

As mentioned previously, the electro-thermo-elastic governing equations of the FGP cylinder, for each harmonic $k$, can now be discretized using FEM. This procedure is shown in the flowchart depicted in Figure 2, and it is explained in the sequel.

\subsubsection{Hierarchical discretization}

The main idea of this section is to introduce a set of hierarchic $p$-order shape functions so that they can be used in a hierarchic finite element. In an auxiliary index space $\Re(\Omega)$ with $\Omega:=\{\xi \mid-1 \leq \xi \leq 1\}$, two shape functions associated to element sides at $\xi= \pm 1$ are defined as [47]: 


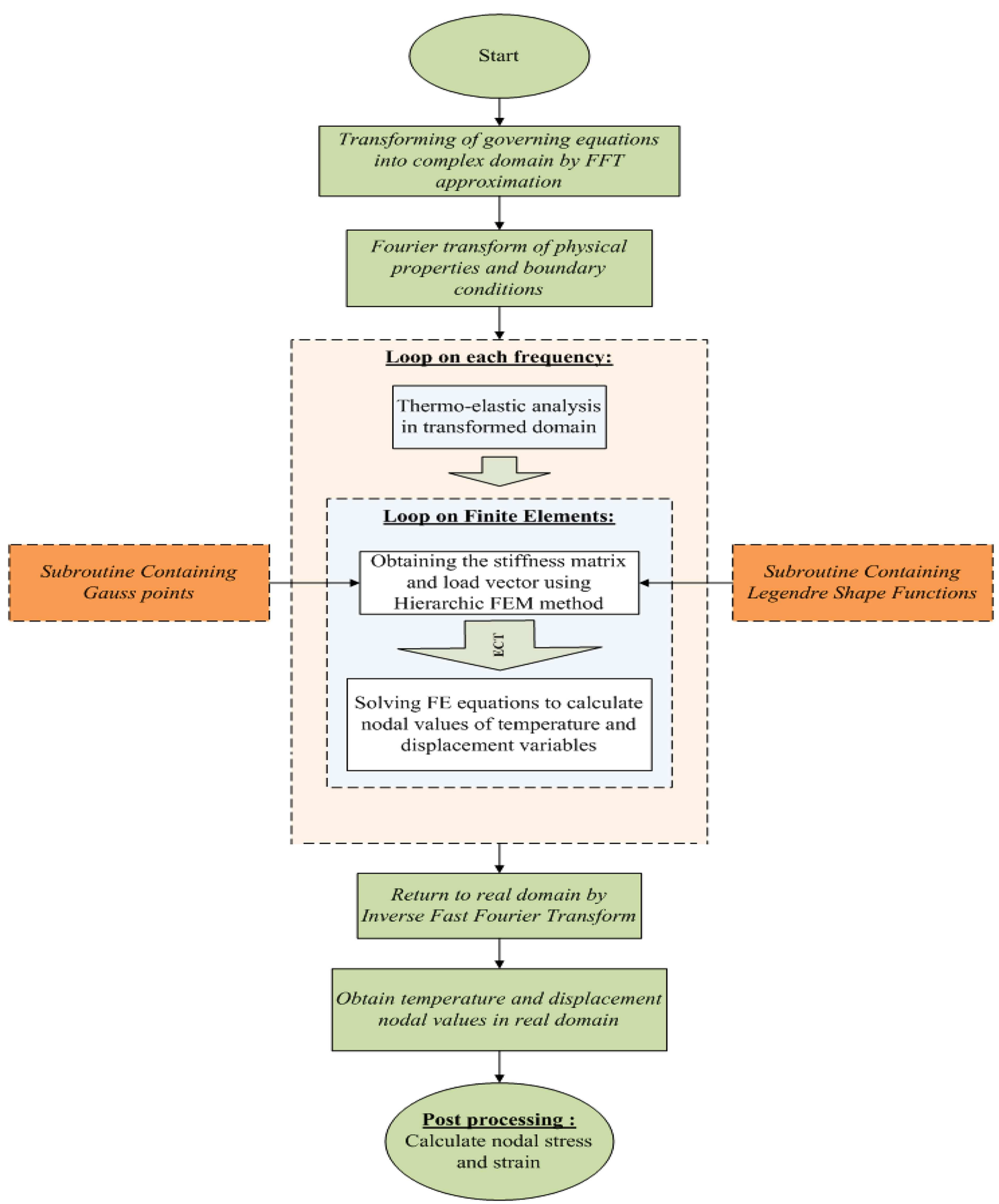

Figure 2. FFT- $p$ FE analysis flowchart.

$$
\hat{\psi}_{1}(\xi)=\frac{1-\xi}{2}, \quad \hat{\psi}_{2}(\xi)=\frac{1+\xi}{2}, \quad \xi \in \Omega
$$

and the other shape functions associated with element region at interval $-1<\xi<1$ are:

$$
\hat{\psi}_{p+1}=\int N_{p}(\xi) \mathrm{d} \xi, \quad \xi \in \Omega, \quad p>1
$$

where:

$$
N_{p}(\xi)=\frac{1}{(p-1) !} \frac{1}{2^{p-1}} \frac{d^{p}}{d \xi^{p}}\left[\left(\xi^{2}-1\right)^{p}\right],
$$

in which $N_{p}(\xi)$ shows the Legendre polynomial in which subscript $p$ indicates the degree of the related polynomials. Now, unknown variables of the problem 
are approximated using the aforementioned shape functions:

$$
\begin{aligned}
& \left\{\begin{array}{l}
\hat{u}_{r}^{k} \\
\hat{u}_{\theta}^{k} \\
\hat{\varphi}^{k}
\end{array}\right\}^{e} \\
& =\left[\begin{array}{ccc}
{\left[\hat{\psi}_{1} \ldots \hat{\psi}_{p+1}\right]} & 0 \\
0 & {\left[\hat{\psi}_{1} \ldots \hat{\psi}_{p+1}\right]} & 0 \\
0 & {\left[\hat{\psi}_{1} \ldots \hat{\psi}_{p+1}\right]}
\end{array}\right]
\end{aligned}
$$

$$
\left\{\begin{array}{c}
\left\{\hat{u}_{r}\right\} \\
\left\{\hat{u}_{\theta}\right\} \\
\{\hat{\varphi}\}
\end{array}\right\}^{e}
$$

where:

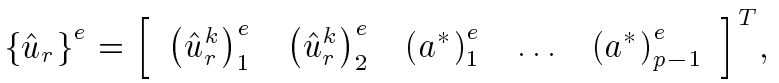

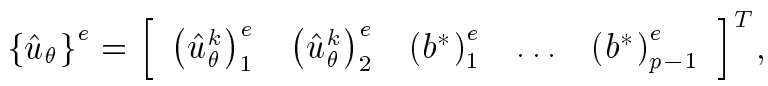

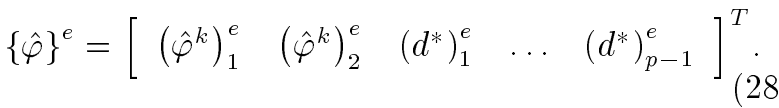

The coefficients $\left(a^{*}\right)_{i}^{e},\left(b^{*}\right)_{i}^{e}$ and $\left(d^{*}\right)_{i}^{e}, i=1, \ldots, p-1$ are auxiliary parameters of a hierarchical finite element that can be eliminated using the Element Condensation (EC) technique. Substituting approximation functions of Eq. (27) into the governing Eqs. (20)-(22), gives the following set of equations in the matrix form:

$$
\left[\begin{array}{ll}
{\left[\hat{K}_{11}^{e}\right]} & {\left[\hat{K}_{12}^{e}\right]} \\
{\left[\hat{K}_{21}^{e}\right]} & {\left[\hat{K}_{22}^{e}\right]}
\end{array}\right]\left\{\begin{array}{l}
\{\hat{U}\}^{e} \\
\left\{D^{*}\right\}^{e}
\end{array}\right\}=\left\{\begin{array}{l}
\left\{\hat{F}_{1}^{e}\right\} \\
\left\{\hat{F}_{2}^{e}\right\}
\end{array}\right\},
$$

where:

$$
\left\{\begin{array}{l}
\left\{\hat{F}_{1}^{e}\right\} \\
\left\{\hat{F}_{2}^{e}\right\}
\end{array}\right\}=\left\{\begin{array}{l}
\left\{\hat{f}_{1}^{e}\right\} \\
\left\{\hat{f}_{2}^{e}\right\}
\end{array}\right\}-\left[\begin{array}{ll}
{\left[\hat{G}_{11}^{e}\right]} & {\left[\hat{G}_{12}^{e}\right]} \\
{\left[\hat{G}_{21}^{e}\right]} & {\left[\hat{G}_{22}^{e}\right]}
\end{array}\right]\left\{\begin{array}{l}
\{\hat{T}\} \\
\{\hat{T}\}
\end{array}\right\},
$$

and:

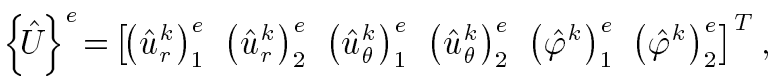

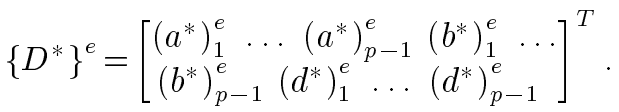

Using the EC technique in the absence of auxiliary parameters, the assembly procedure is performed to obtain the imaginary unknown variables in the frequency space. So, we have:

$$
\left[\hat{K}^{*}\right]\{\hat{U}\}=\left\{\hat{F}^{*}\right\}
$$

where:

$$
\begin{aligned}
& {\left[\hat{K}^{*}\right]=\left(\left[\hat{K}_{11}\right]-\left[\hat{K}_{12}\right]\left[\hat{K}_{22}\right]^{-1}\left[\hat{K}_{21}\right]\right),} \\
& \left\{\hat{F}^{*}\right\}=\left(\left\{\hat{F}_{1}\right\}-\left[\hat{K}_{12}\right]\left[\hat{K}_{22}\right]^{-1}\left\{\hat{F}_{2}\right\}\right) .
\end{aligned}
$$

Since the results obtained from this section are in frequency space, it is necessary to invert them into the physical space. The inverse FFT technique can be effectively used for this aim. In the present investigation, the FFTW3 library is used for the required FFT and inverse FFT.

\section{3D discretization of the FGP hollow cylinder using higher-order elements}

\subsection{D heat conduction problem}

In this section, the ability of the proposed FFTFE method to analyze the three-dimensional heat conduction of a thick hollow cylinder (Figure 3) under asymmetric thermal excitation is investigated. A review of the literature over the past few decades shows that there are few finite element solutions to the heat conduction problem in cylindrical or spherical coordinate systems [48]. In this study, both the geometry and the governing equations are discretized in the cylindrical coordinate system using FFT- $p$ FE method. For this purpose, three-dimensional heat equation in cylindrical coordinate and in the absence of heat generation is considered as follows [8]:

$$
\begin{aligned}
& \lambda_{r} \frac{\partial^{2} T}{\partial r^{2}}+\frac{1}{r} \lambda_{r} \frac{\partial T}{\partial r}+\lambda_{r} \frac{1}{r^{2}} \frac{\partial^{2} T}{\partial \theta^{2}}+\frac{d \lambda_{r}}{d r} \frac{\partial T}{\partial r} \\
&+\lambda_{r} \frac{\partial^{2} T}{\partial z^{2}}=0, \\
& a \leq r \leq b, \quad 0<\theta \leq 2 \pi, \quad 0 \leq z \leq h,
\end{aligned}
$$

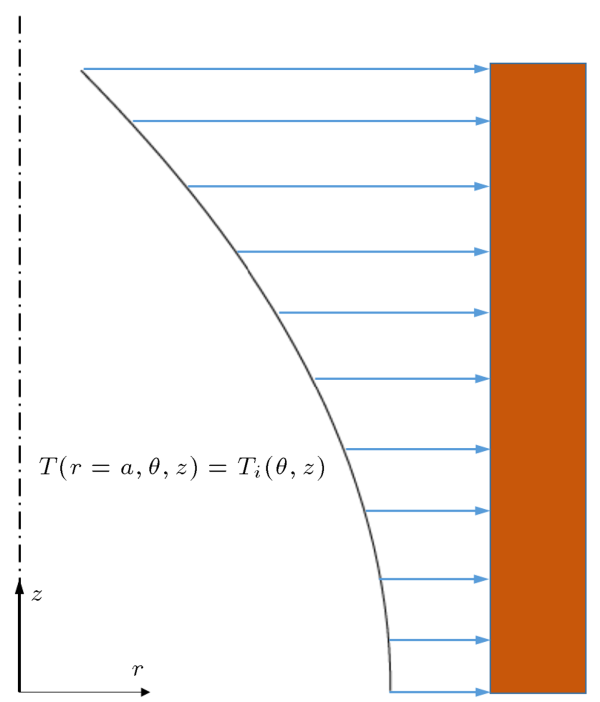

Figure 3. Variation of the temperature field along the cylinder axis. 


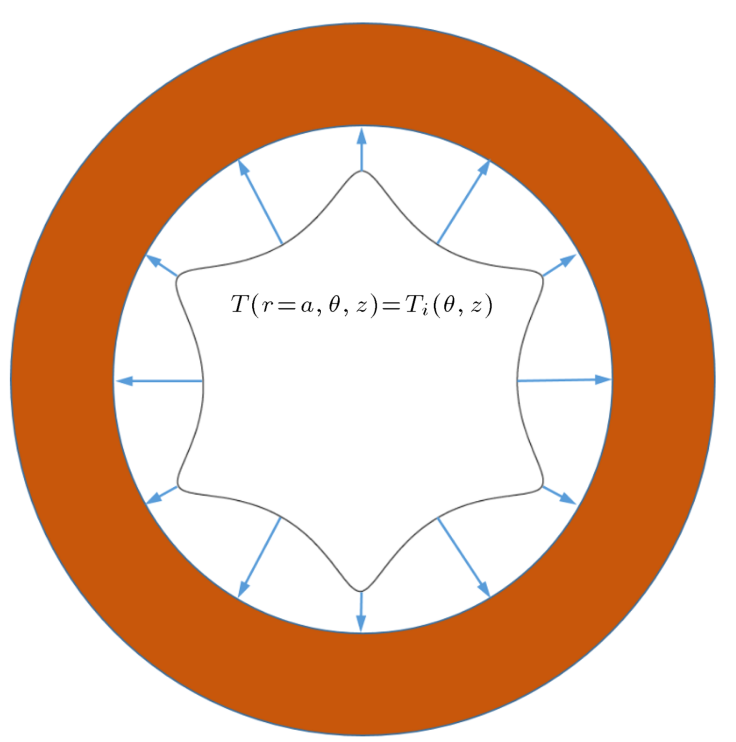

Figure 4. Circumferential distribution of the temperature field.

where $\lambda_{r}$ is defined as Eq. (3). As shown in Figure 4, the relevant thermal boundary conditions of the inner and outer surfaces of the cylinder are considered to have the following form:

$$
\begin{aligned}
& T(r=a, z, \theta)=T_{i}(z, \theta), \quad T(r=b, z, \theta)=0, \\
& q_{n}(r, z=0, \theta)=0, \quad q_{n}(r, z=h, \theta)=0,
\end{aligned}
$$

where $q_{n}$ is heat input (or output) into (or from) the two ends of the cylinder, $T$ is the temperature field relative to the reference temperature and $T_{i}$ is the distribution of the temperature across the inner surface of the cylinder.

Now, using the weighted residual method, the weak form of the above heat equation can be expressed as:

$$
\begin{gathered}
\int_{\Omega^{e}}\left[\frac{\partial \mathbf{w}}{\partial r}\left(\lambda_{r} \frac{\partial T}{\partial r}\right)+\frac{\partial \mathbf{w}}{\partial z}\left(\lambda_{r} \frac{\partial T}{\partial z}\right)-\frac{\mathbf{w}}{r^{2}} \frac{\partial^{2} T}{\partial \theta^{2}}\right] \\
r d r d z d \theta-\int_{\Gamma^{e}} \mathbf{w} q_{n} d s=0
\end{gathered}
$$

where:

$$
q_{n}=\left(\lambda_{r} \frac{\partial T}{\partial r} n_{r}+\lambda_{z} \frac{\partial T}{\partial z} n_{z}+\lambda_{\theta} \frac{1}{r} \frac{\partial T}{\partial \theta} n_{\theta}\right)
$$

in which, w is a vector of weight functions from the Lagrange family used for interpolating the field variable, $T$, and heat flux, $q_{n}$. Mutually, the discrete Fourier transform technique is well used to discretize heat equation in the circumferential direction. A set of approximate functions, including Fourier coefficients of the field variable, are substituted in the above equation.
As mentioned previously, the field variable is defined at discrete points $\theta_{K}, k=1,2, \ldots, N$, where $k$ is known as the wave number. The following relation is then introduced for interpolating the temperature variable:

$$
T(r, \theta, z)=\sum_{k=1}^{N} \hat{T}_{k}(r, z) e^{i k \theta}
$$

By substituting this relation into the Eq. (36), the residual heat equation in terms of the radial and axial variables becomes:

$$
\begin{gathered}
\int_{\Omega^{e}}\left[\frac{\partial \mathbf{w}}{\partial r}\left(\lambda_{r} \frac{\partial \hat{T}_{k}}{\partial r}\right)+\frac{\partial \mathbf{w}}{\partial z}\left(\lambda_{r} \frac{\partial \hat{T}_{k}}{\partial z}\right)+\frac{\mathbf{w} k^{2}}{r^{2}} \hat{T}_{k}\right] \\
r d r d z=0 \text { for } k=1, \ldots, N .
\end{gathered}
$$

Related boundary conditions are as follows:

$$
\begin{cases}\hat{T}\left(r=a, z, \theta_{k}\right)=\hat{T}_{i}\left(z, \theta_{k}\right), & \\ \hat{T}\left(r=b, z, \theta_{k}\right)=0, & \text { For } k=1, \ldots, N \\ \hat{q}\left(r, z=0, \theta_{k}\right)=0, & \\ \hat{q}\left(r, z=h, \theta_{k}\right)=0 . & \end{cases}
$$

It should be noted that in the above equation, the strong form of the spatial derivative with respect to the circumferential variable should be retained. Now, the meridian cross-section of the cylinder can be discretized using finite elements, including $N_{r}$ nodes in each harmonic. 2D rectangular linear and higherorder elements from the Lagrange family are used to discretize the geometry and for the heat equation, respectively.

In each element, $\hat{T}_{k}(r, z)$ is approximated by the interpolation functions, $\chi_{j}^{e}$, so we have:

$$
\hat{T}_{k}^{e}(r, z)=\sum_{j=1}^{N_{r}} \chi_{j}^{e}(r, z)\left(\hat{T}_{k}^{e}\right)_{j}
$$

where $\left(\hat{T}_{k}^{e}\right)_{j}$ are nodal values corresponding to the temperature field in the Fourier space. According to the Galerkin method, the weight functions, $w_{i}$, are considered to be the same as the interpolation functions. In the computation of the weighted integrals, a local coordinate system $(\xi, \eta)$ is defined for the sake of simplicity. For this, we need to make a proper mapping between the parametric space and the physical space. $r, z$ variables in terms of the local coordinate parameters $(\xi, \eta)$ as well as the transformation Jacobian matrix can be defined as:

$$
\begin{aligned}
\left\{\begin{array}{l}
r \\
z
\end{array}\right\} & =\left\{\begin{array}{l}
\sum_{j=1}^{m} r_{j}^{e} \chi_{j}^{e}(\xi) \\
\sum_{j=1}^{m} z_{j}^{e} \chi_{j}^{e}(\xi)
\end{array}\right\} \\
& =\left[\begin{array}{llll}
r_{1} & r_{2} & \ldots & r_{m} \\
z_{1} & z_{2} & \ldots & z_{m}
\end{array}\right]\left[\begin{array}{llll}
\chi_{1} & \chi_{2} & \cdots & \chi_{m}
\end{array}\right]^{T}
\end{aligned}
$$



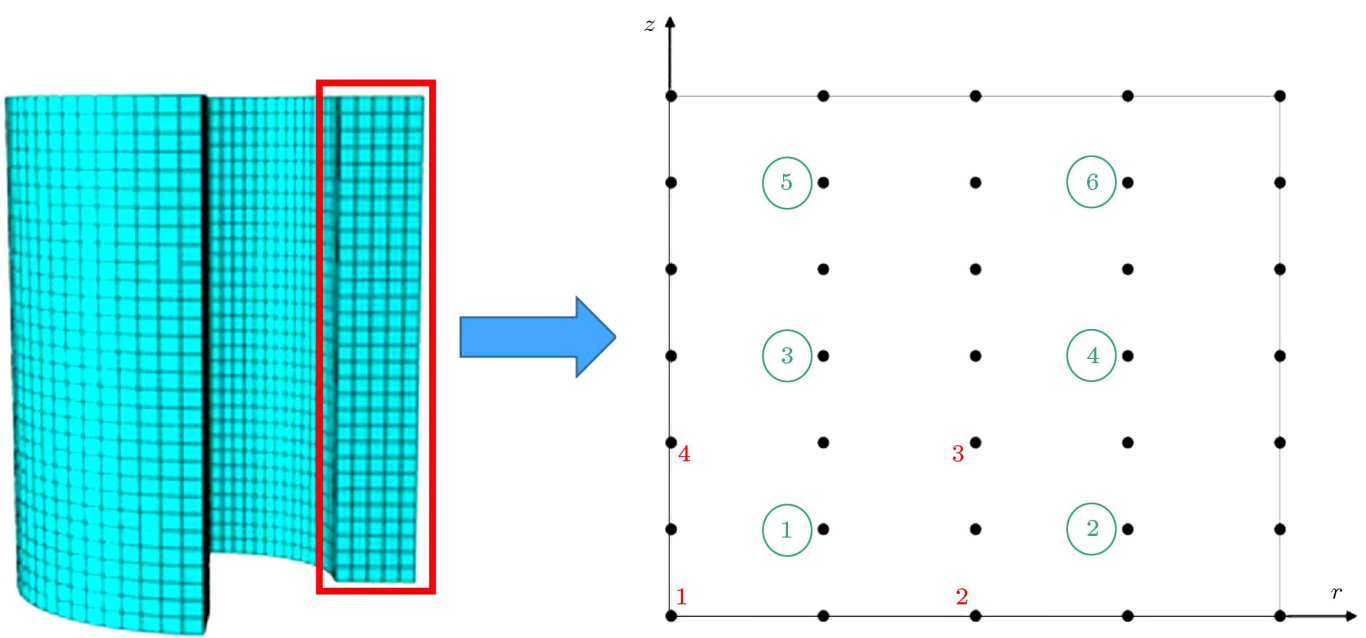

Figure 5. Discretized model of the cylinder.

$$
\left\{\begin{array}{l}
\frac{\partial T}{\partial \xi} \\
\frac{\partial T}{\partial \eta}
\end{array}\right\}=\left[\begin{array}{ll}
\frac{\partial r}{\partial \xi} & \frac{\partial z}{\partial \xi} \\
\frac{\partial r}{\partial \eta} & \frac{\partial z}{\partial \eta}
\end{array}\right]\left\{\begin{array}{c}
\frac{\partial T}{\partial r} \\
\frac{\partial T}{\partial z}
\end{array}\right\}
$$

Using this geometrical approximation, we can solve the governing equation of the problem. As we considered in the previous section, the Gauss-Legendre method is used for numerical computing of the stiffness matrix. Discretized model of the thick hollow cylinder is shown in Figure 5. Discretized heat conduction equation in the matrix form yields,

$$
[\hat{K}]\left\{\hat{T}_{k}\right\}=\{\hat{Q}\} \text {. }
$$

\subsection{Thermo-electro-elastic demonstration}

In this section, the previous FFT- $p$ FE methodology is considered for three-dimensional thermo-electro-elastic analysis of an FGP hollow cylinder. In spite of the previous $2 \mathrm{D}$ discretization, the classical second-order finite elements with Lagrangian interpolation functions are used in the 3D discretization. Accordingly, we deal with a FG hollow cylinder (Figure 3), and the kinematic relationship between its strain and displacement components (in cylindrical coordinates) are as follows:

$$
\begin{aligned}
& \varepsilon_{r r}=\frac{\partial u_{r}}{\partial r}, \quad \varepsilon_{\theta \theta}=\frac{1}{r}\left(u_{r}+\frac{\partial u_{\theta}}{\partial \theta}\right), \quad \varepsilon_{z z}=\frac{\partial u_{z}}{\partial z}, \\
& \varepsilon_{r \theta}=\frac{1}{2}\left(\frac{\partial u_{r}}{r \partial \theta}+\frac{\partial u_{\theta}}{\partial r}-\frac{u_{\theta}}{r}\right), \\
& \varepsilon_{\theta z}=\frac{1}{2}\left(\frac{\partial u_{\theta}}{\partial z}+\frac{\partial u_{z}}{r \partial \theta}\right), \\
& \varepsilon_{z r}=\frac{1}{2}\left(\frac{\partial u_{z}}{\partial r}+\frac{\partial u_{r}}{\partial z}\right), \\
& E_{r}=-\frac{\partial \varphi}{\partial r}, \quad E_{\theta}=-\frac{\partial \varphi}{r \partial \theta}, \quad E_{z}=-\frac{\partial \varphi}{\partial z} .
\end{aligned}
$$

The generalized constitutive relations of orthotropicpiezoelectric materials polarized in the axial direction are considered as below [19]:

$$
\begin{aligned}
& \sigma_{r r}=C_{11} \varepsilon_{r r}+C_{12} \varepsilon_{\theta \theta}+C_{13} \varepsilon_{z z}-\beta_{1} T-e_{31} E_{z}, \\
& \sigma_{\theta z}=2 C_{44} \varepsilon_{\theta z}-e_{24} E_{\theta}, \\
& \sigma_{\theta \theta}=C_{12} \varepsilon_{r r}+C_{22} \varepsilon_{\theta \theta}+C_{23} \varepsilon_{z z}-\beta_{2} T-e_{32} E_{z}, \\
& \sigma_{r z}=2 C_{55} \varepsilon_{r z}-e_{15} E_{r}, \\
& \sigma_{z z}=C_{13} \varepsilon_{r r}+C_{23} \varepsilon_{\theta \theta}+C_{33} \varepsilon_{z z}-\beta_{3} T-e_{33} E_{z}, \\
& \sigma_{r \theta}=2 C_{66} \varepsilon_{r \theta}, \\
& D_{r}=2 e_{15} \varepsilon_{r z}+\eta_{11} E_{r}+\mathrm{P}_{1} T, \\
& D_{\theta}=2 e_{24} \varepsilon_{\theta z}+\eta_{22} E_{\theta}+\mathrm{P}_{2} T, \\
& D_{z}=2\left(e_{31} \varepsilon_{r r}+e_{32} \varepsilon_{\theta \theta}+e_{33} \varepsilon_{z z}\right)+\eta_{33} E_{z}+\mathrm{P}_{3} T,
\end{aligned}
$$

where:

$$
\left\{\begin{array}{l}
\beta_{1}=C_{11} \alpha_{r}+C_{12} \alpha_{\theta}+C_{13} \alpha_{z}, \\
\beta_{2}=C_{12} \alpha_{r}+C_{22} \alpha_{\theta}+C_{23} \alpha_{z}, \\
\beta_{3}=C_{13} \alpha_{r}+C_{23} \alpha_{\theta}+C_{33} \alpha_{z} .
\end{array}\right.
$$

Similarly, Hamilton's principle of Eq. (12) is used to derive the governing equations of the FGP hollow cylinder. Considering these approximation functions for the field variables of the thermo-electro-elastic problem:

$$
\begin{aligned}
& u_{r}(r, \theta, z)=\sum_{k=1}^{N} \sum_{j=1}^{N_{r}} \chi_{j}(r, z)\left(\hat{u}_{r}\right)_{j}^{k} e^{i k \theta}, \\
& u_{\theta}(r, \theta, z)=\sum_{k=1}^{N} \sum_{j=1}^{N_{r}} \chi_{j}(r, z)\left(\hat{u}_{\theta}\right)_{j}^{k} e^{i k \theta},
\end{aligned}
$$




$$
\begin{aligned}
& u_{z}(r, \theta, z)=\sum_{k=1}^{N} \sum_{j=1}^{N_{r}} \chi_{j}(r, z)\left(\hat{u}_{z}\right)_{j}^{k} e^{i k \theta}, \\
& \varphi(r, \theta, z)=\sum_{k=1}^{N} \sum_{j=1}^{N_{r}} \chi_{j}(r, z)(\hat{\varphi})_{j}^{k} e^{i k \theta},
\end{aligned}
$$

and substituting kinematic and constitutive relations of Eqs. (45) and (46) into the prescribed energy method, the following four sets of discretized governing equations at each harmonic $k$ with $k=1, \ldots, N$ yields:

$$
\begin{aligned}
& \left(\delta \hat{u}_{r}\right)_{i}^{k}: \sum_{j=1}^{N_{r}} \bar{D}_{11}^{i j}\left(\hat{u}_{r}\right)_{j}^{k}+\sum_{j=1}^{N_{r}} \bar{A}_{12}^{i j}\left(\hat{u}_{r}\right)_{j}^{k} \\
& +\sum_{j=1}^{N_{r}} \bar{G}_{12}^{i j}\left(\left(\hat{u}_{r}\right)_{j}^{k}+i k\left(\hat{u}_{\theta}\right)_{j}^{k}\right) \\
& +\sum_{j=1}^{N_{r}} \bar{B}_{22}^{i j}\left(\left(\hat{u}_{r}\right)_{j}^{k}+i k\left(\hat{u}_{\theta}\right)_{j}^{k}\right) \\
& +\sum_{j=1}^{N_{r}} \bar{H}_{55}^{i j}\left(\hat{u}_{r}\right)_{j}^{k}+\sum_{j=1}^{N_{r}} \bar{L}_{55}^{i j}\left(\hat{u}_{z}\right)_{j}^{k} \\
& +k^{2} \sum_{j=1}^{N_{r}} \bar{B}_{66}^{i j}\left(\hat{u}_{r}\right)_{j}^{k}-i k \\
& \left(\sum_{j=1}^{N_{r}} \bar{A}_{66}^{i j}\left(\hat{u}_{r}\right)_{j}^{k}-\sum_{j=1}^{N_{r}} \bar{B}_{66}^{i j}\left(\hat{u}_{r}\right)_{j}^{k}\right) \\
& +\sum_{j=1}^{N_{r}} \bar{I}_{13}^{i j}\left(\hat{u}_{z}\right)_{j}^{k}+\sum_{j=1}^{N_{r}} \bar{J}_{23}^{i j}\left(\hat{u}_{z}\right)_{j}^{k} \\
& +\sum_{j=1}^{N_{r}} \tilde{L}_{15}^{i j} \hat{\varphi}_{j}^{k}+\sum_{j=1}^{N_{r}} \tilde{I}_{31}^{i j} \hat{\varphi}_{j}^{k}+\sum_{j=1}^{N_{r}} \tilde{J}_{32}^{i j} \hat{\varphi}_{j}^{k} \\
& -\sum_{j=1}^{N_{r}} \bar{F}_{1}^{i j} \hat{T}_{j}^{k}+\sum_{j=1}^{N_{r}} \bar{E}_{2}^{i j} \hat{T}_{j}^{k}=0 .
\end{aligned}
$$

$$
\begin{aligned}
\left(\delta \hat{u}_{\theta}\right)_{i}^{k}: & -i k \sum_{j=1}^{N_{r}} \bar{A}_{12}^{i j}\left(\hat{u}_{r}\right)_{j}^{k}-\sum_{j=1}^{N_{r}} \bar{B}_{22}^{i j}\left(i k\left(\hat{u}_{r}\right)_{j}^{k}-k^{2}\left(\hat{u}_{\theta}\right)_{j}^{k}\right) \\
& -\sum_{j=1}^{N_{r}} \bar{H}_{44}^{i j}\left(\hat{u}_{\theta}\right)_{j}^{k}+i k \sum_{j=1}^{N_{r}} \bar{M}_{44}^{i j}\left(\hat{u}_{z}\right)_{j}^{k} \\
& -i k \sum_{j=1}^{N_{r}} \bar{J}_{23}^{i j}\left(\hat{u}_{z}\right)_{j}^{k}+\sum_{j=1}^{N_{r}} \bar{G}_{66}^{i j}\left(i k\left(\hat{u}_{r}\right)_{j}^{k}-\left(\hat{u}_{\theta}\right)_{j}^{k}\right)
\end{aligned}
$$

$$
\begin{aligned}
& -\sum_{j=1}^{N_{r}} \bar{B}_{66}^{i j}\left(\left(\hat{u}_{r}\right)_{j}^{k}-\left(\hat{u}_{\theta}\right)_{j}^{k}\right)+\sum_{j=1}^{N_{r}} \bar{D}_{66}^{i j}\left(\hat{u}_{\theta}\right)_{j}^{k} \\
& +\sum_{j=1}^{N_{r}} \bar{A}_{66}^{i j}\left(\hat{u}_{\theta}\right)_{j}^{k}-i k \sum_{j=1}^{N_{r}} \tilde{J}_{32}^{i j} \hat{\varphi}_{j}^{k} \\
& +i k \sum_{j=1}^{N_{r}} \tilde{M}_{24}^{i j} \hat{\varphi}_{j}^{k}+i k \sum_{j=1}^{N_{r}} \bar{E}_{2}^{i j} \hat{T}_{j}^{k}=0,
\end{aligned}
$$

$$
\begin{aligned}
\left(\delta \hat{u}_{z}\right)_{i}^{k}: & \sum_{j=1}^{N_{r}} \bar{L}_{13}^{i j}\left(\hat{u}_{r}\right)_{j}^{k}+\sum_{j=1}^{N_{r}} \bar{M}_{23}^{i j}\left(\left(\hat{u}_{r}\right)_{j}^{k}+i k\left(\hat{u}_{\theta}\right)_{j}^{k}\right) \\
& +\sum_{j=1}^{N_{r}}\left(\bar{I}_{55}^{i j}\left(\hat{u}_{r}\right)_{j}^{k}+D_{55}^{i j}\left(\hat{u}_{z}\right)_{j}^{k}\right) \\
& +\sum_{j=1}^{N_{r}}\left(-i k \bar{J}_{44}^{i j}\left(\hat{u}_{\theta}\right)_{j}^{k}+k^{2} \bar{B}_{44}^{i j}\left(\hat{u}_{z}\right)_{j}^{k}\right) \\
& +\sum_{j=1}^{N_{r}} \bar{H}_{33}^{i j}\left(\hat{u}_{z}\right)_{j}^{k}+\sum_{j=1}^{N_{r}} \tilde{H}_{33}^{i j} \hat{\varphi}_{j}^{k}+k^{2} \sum_{j=1}^{N_{r}} \tilde{B}_{24}^{i j} \hat{\varphi}_{j}^{k} \\
& +\sum_{j=1}^{N_{r}} \tilde{D}_{15}^{i j} \hat{\varphi}_{j}^{k}-\sum_{j=1}^{N_{r}} \bar{N}_{3} \hat{T}_{j}^{k}=0,
\end{aligned}
$$$$
\delta \hat{\varphi}_{i}^{k}: \sum_{j=1}^{N_{r}}\left(\tilde{I}_{15}^{i j}\left(\hat{u}_{r}\right)_{j}^{k}+\tilde{D}_{15}^{i j}\left(\hat{u}_{z}\right)_{j}^{k}\right)
$$$$
+\sum_{j=1}^{N_{r}} \tilde{M}_{32}^{i j}\left(\left(\hat{u}_{r}\right)_{j}^{k}+i k\left(\hat{u}_{\theta}\right)_{j}^{k}\right)+\sum_{j=1}^{N_{r}} \tilde{L}_{31}^{i j}\left(\hat{u}_{r}\right)_{j}^{k}
$$$$
+\sum_{j=1}^{N_{r}}\left(k^{2} \tilde{B}_{24}^{i j}\left(\hat{u}_{z}\right)_{j}^{k}-i k \tilde{J}_{24}^{i j}\left(\hat{u}_{\theta}\right)_{j}^{k}\right)
$$$$
+\sum_{j=1}^{N_{r}} \tilde{H}_{33}^{i j}\left(\hat{u}_{z}\right)_{j}^{k}-\sum_{j=1}^{N_{r}} \widehat{D}^{i j}{ }_{11} \hat{\varphi}_{j}^{k}-k^{2} \sum_{j=1}^{N_{r}} \widehat{B}_{22}^{i j} \hat{\varphi}_{j}^{k}
$$$$
-\sum_{j=1}^{N_{r}} \widehat{H}_{33}^{i j} \hat{\varphi}_{j}^{k}+\sum_{j=1}^{N_{r}} \tilde{F}_{1}^{i j} \hat{T}_{j}^{k}-i k \sum_{j=1}^{N_{r}} \tilde{E}_{2}^{i j} \hat{T}_{j}^{k}
$$$$
+\sum_{j=1}^{N_{r}} \tilde{N}_{3}^{i j} \hat{T}_{j}^{k}=0
$$

where:

$$
\bar{A}_{m n}^{i j}=\iint_{\Omega^{e}} C_{m n} \chi_{i}\left(\frac{\partial \chi_{j}}{\partial r}\right) d r d z
$$




$$
\begin{aligned}
& \bar{B}_{m n}^{i j}=\iint_{\Omega^{e}} C_{m n}\left(\frac{1}{r}\right) \chi_{i} \chi_{j} d r d z \\
& \bar{D}_{m n}^{i j}=\iint_{\Omega^{e}} C_{m n}\left(\frac{\partial \chi_{i}}{\partial r}\right)\left(\frac{\partial \chi_{j}}{\partial r}\right) r d r d z, \\
& \bar{G}_{m n}^{i j}=\iint_{\Omega^{e}} C_{m n}\left(\frac{\partial \chi_{i}}{\partial r}\right) \chi_{j} d r d z \\
& \bar{H}_{m n}^{i j}=\iint_{\Omega^{e}} C_{m n}\left(\frac{\partial \chi_{i}}{\partial z}\right)\left(\frac{\partial \chi_{j}}{\partial z}\right) r d r d z, \\
& \bar{I}_{m n}^{i j}=\iint_{\Omega^{e}} C_{m n}\left(\frac{\partial \chi_{i}}{\partial r}\right)\left(\frac{\partial \chi_{j}}{\partial z}\right) r d r d z \\
& \bar{J}_{m n}^{i j}=\iint_{\Omega^{e}} C_{m n} \chi_{i}\left(\frac{\partial \chi_{j}}{\partial z}\right) d r d z \\
& \bar{L}_{m n}^{i j}=\iint_{\Omega^{e}} C_{m n}\left(\frac{\partial \chi_{i}}{\partial z}\right)\left(\frac{\partial \chi_{j}}{\partial r}\right) r d r d z, \\
& \tilde{E}_{m}^{i j}=\iint_{\Omega^{e}} \mathrm{P}_{m} \chi_{i} \chi_{j} d r d z \\
& \tilde{F}_{m}^{i j}=\iint_{\Omega^{e}} \mathrm{P}_{m}\left(\frac{\partial \chi_{i}}{\partial r}\right) \chi_{j} r d r d z \\
& \tilde{N}_{m}^{i j}=\iint_{\Omega^{e}} \mathrm{P}_{m}\left(\frac{\partial \chi_{i}}{\partial z}\right) \chi_{j} r d r d z, \\
& \widehat{D}_{m n}^{i j}=\iint_{\Omega^{e}} \eta_{m n}\left(\frac{\partial \chi_{i}}{\partial r}\right)\left(\frac{\partial \chi_{j}}{\partial r}\right) r d r d z, \\
& \widehat{B}_{m n}^{i j}=\iint_{\Omega^{e}} \eta_{m n}\left(\frac{1}{r}\right) \chi_{i} \chi_{j} d r d z \\
& \widehat{H}_{m n}^{i j}=\iint_{\Omega^{e}} \eta_{m n}\left(\frac{\partial \chi_{i}}{\partial z}\right)\left(\frac{\partial \chi_{j}}{\partial z}\right) r d r d z, \\
& \tilde{I}_{m n}^{i j}=\iint_{\Omega^{e}} e_{m n}\left(\frac{\partial \chi_{i}}{\partial r}\right)\left(\frac{\partial \chi_{j}}{\partial z}\right) r d r d z, \\
& \tilde{D}_{m n}^{i j}=\iint_{\Omega^{e}} e_{m n}\left(\frac{\partial \chi_{i}}{\partial r}\right)\left(\frac{\partial \chi_{j}}{\partial r}\right) r d r d z, \\
& \tilde{M}_{m n}^{i j}=\iint_{\Omega^{e}} e_{m n}\left(\frac{\partial \chi_{i}}{\partial z}\right) \chi_{j} d r d z \\
& \tilde{L}_{m n}^{i j}=\iint_{\Omega^{e}} e_{m n}\left(\frac{\partial \chi_{i}}{\partial z}\right)\left(\frac{\partial \chi_{j}}{\partial r}\right) r d r d z \\
& \tilde{B}_{m n}^{i j}=\iint_{\Omega^{e}} e_{m n}\left(\frac{1}{r}\right) \chi_{i} \chi_{j} d r d z
\end{aligned}
$$

$$
\begin{aligned}
& \tilde{J}_{m n}^{i j}=\iint_{\Omega^{e}} e_{m n} \chi_{i}\left(\frac{\partial \chi_{j}}{\partial z}\right) d r d z, \\
& \tilde{H}_{m n}^{i j}=\iint_{\Omega^{e}} e_{m n}\left(\frac{\partial \chi_{i}}{\partial z}\right)\left(\frac{\partial \chi_{j}}{\partial z}\right) r d r d z, \\
& \bar{N}_{m}^{i j}=\iint_{\Omega^{e}} \beta_{m}\left(\frac{\partial \chi_{i}}{\partial z}\right) \chi_{j} r d r d z, \\
& \bar{E}_{m}^{i j}=\iint_{\Omega^{e}} \beta_{m} \chi_{i} \chi_{j} d r d z, \\
& \bar{F}_{m}^{i j}=\iint_{\Omega^{e}} \beta_{m}\left(\frac{\partial \chi_{i}}{\partial r}\right) \chi_{j} r d r d z .
\end{aligned}
$$

Rewriting these governing equations in the matrix form for $k=1, \ldots, N$ yields:

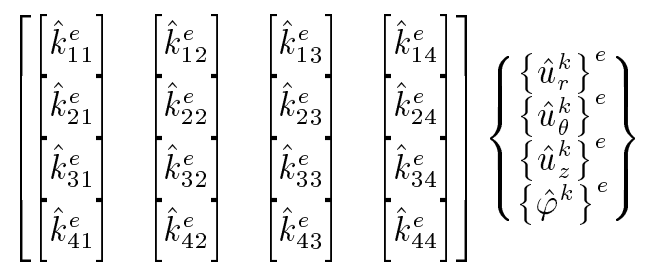

$$
\begin{gathered}
{\left[\begin{array}{cccc}
{\left[\hat{G}_{11}^{e}\right]} & 0 & \cdots & 0 \\
0 & {\left[\hat{G}_{22}^{e}\right]} & \ddots & \vdots \\
\vdots & \ddots & {\left[\hat{G}_{33}^{e}\right]} & 0 \\
0 & \cdots & 0 & {\left[\hat{G}_{44}^{e}\right]}
\end{array}\right]} \\
\left\{\begin{array}{l}
\left\{\hat{T}_{k}^{e}\right\} \\
\left\{\begin{array}{c}
\hat{T}_{k}^{e} \\
\hat{T}_{k}^{e} \\
\hat{T}_{k}^{e}
\end{array}\right\}
\end{array}\right\}=\left\{\begin{array}{l}
\left\{\hat{f}_{1}^{e}\right\} \\
\left\{\begin{array}{l}
\hat{f}_{2}^{e} \\
\hat{f}_{3}^{e}
\end{array}\right\} \\
\left\{\hat{f}_{4}^{e}\right\}
\end{array}\right\} .
\end{gathered}
$$

In the following, we can solve this linear system of equations, formed in the harmonic space, using the FE approach to obtain the results in compliance with the nodal degrees of freedom within the hollow cylinder. Mutually, using the iFFT technique, the harmonic numerical results can be inverted into the real space.

\section{Numerical results}

\subsection{D FFT-pFE results (by hierarchical elements)}

4.1.1. Convergence study

This section aims to illustrate the convergence, accuracy, and effectiveness of the suggested method for the electro-thermo-elastic analysis of an FGP hollow cylinder. Indeed, the solution of the heat conduction 


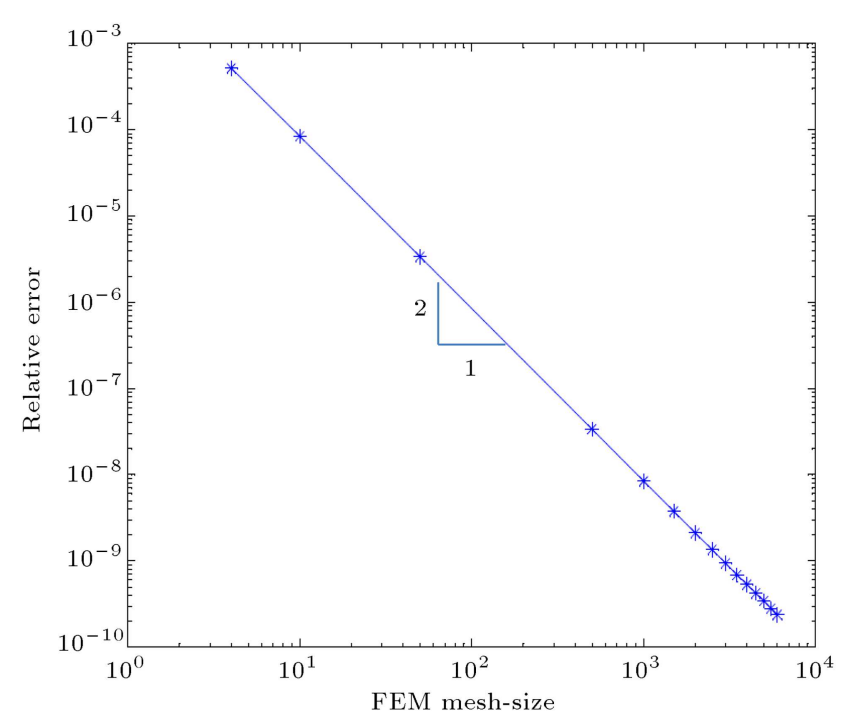

Figure 6. Logarithmic representation of relative error versus the number of finite elements in the $h$-version Finite Element Method (FEM).

equation at the uniform boundary temperature of the inner surface $\left(T_{i}\right)$ and outer surface $\left(T_{o}\right)$ of the cylinder is considered here. In this regard, the following formula can be used for computing the relative error of the obtained results:

$$
\text { Relative error }=\frac{\| \text { Present solution }- \text { Exact solution } \|}{\| \text { Exact solution } \|},
$$

with ||| being an appropriate norm.

Results extracted from the proposed hybrid method are compared with the results of reference [49]. The convergence rate obtained by linear elements is shown in Figure 6 . It is observed that increasing the number of elements in the radial direction is accompanied by a linear convergence behavior of the results. This is known as the $h$-version finite element approach in which the density of linear elements is raised until the desired precision is achieved. On the other hand, convergence is gained in the $p$-version approach using higher-order shape functions. Figure 7 shows a super algebraic convergence behavior of the FFT- $p$ FE method while increasing the order of the hierarchical shape function. Furthermore, the effect of element density on the distribution of various elements is also presented.

In Figure 8, the convergence behavior of the Fourier spectral method is shown for some periodic functions. These functions are assumed to be boundary conditions of the inner surface of the cylinder. As shown in this figure, the rate of convergence varies with the complexity of the periodic functions. As expected, for $T(\theta)=\sin \theta$, the desired method can appropriately attain machine precision in finite harmonics. It is obvious from the figure that the FFT technique has an

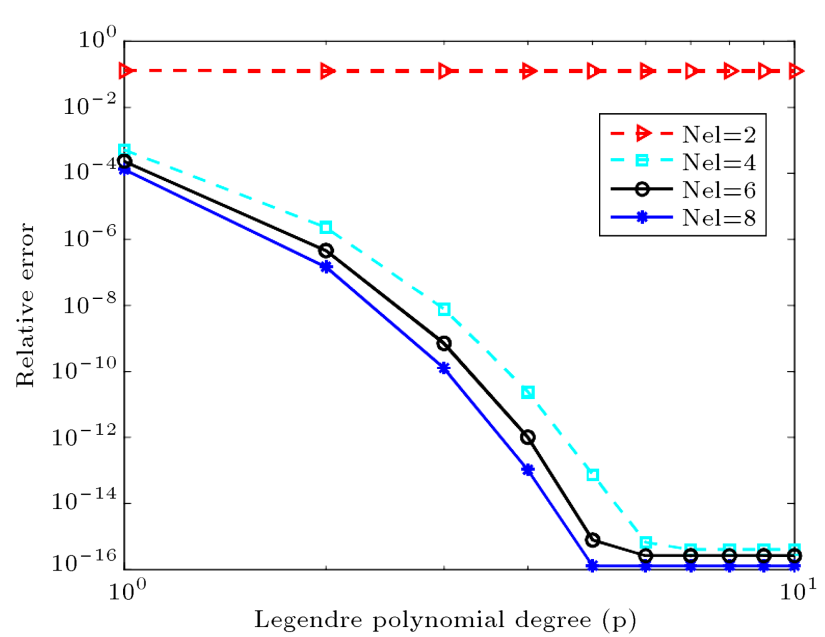

Figure 7. Variation of the relative error versus Legendre polynomial degree.

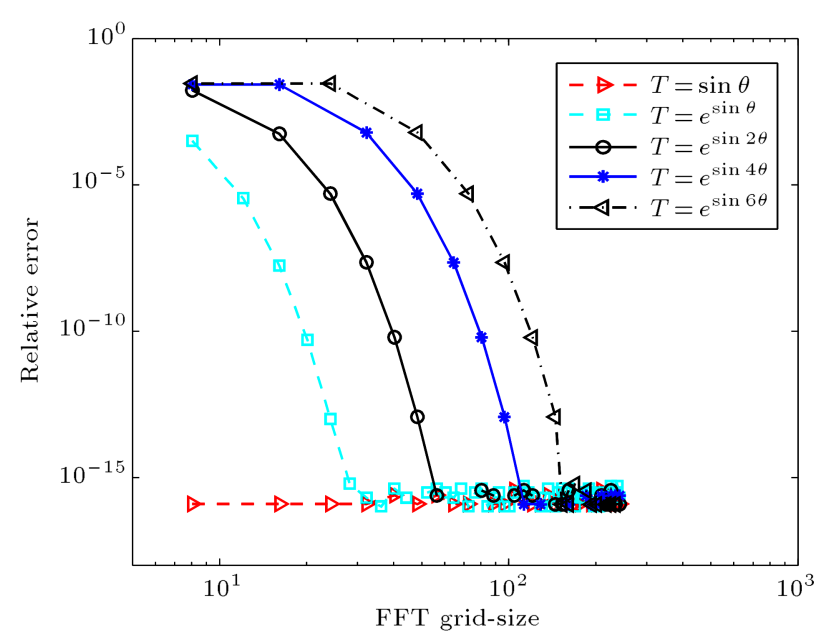

Figure 8. Logarithmic representation of the relative error versus the number of wavenumbers in the Fast Fourier Transform (FFT) technique.

exponentially varying convergence behavior. A comprehensive review revealed that FFT is more vigorous and accurate than FEM for analyzing the problems with a periodic domain.

\subsubsection{Asymmetric demonstration}

In this section, at first, the results of the FGP cylinder under thermal loading are verified using existing literature [5]. For this purpose, the axisymmetric exact solution of an FGP cylinder is extracted. To obtain the results, the properties of the cadmium selenide as an FGP material are used, as given below [5]:

$$
\begin{aligned}
& \alpha_{r}^{0}=2.458 \times 10^{-6} 1 / \mathrm{K}, \\
& \alpha_{\theta}^{0}=4.396 \times 10^{-6} 1 / \mathrm{K}, \quad C_{11}^{0}=83.6 \mathrm{GPa}, \\
& C_{12}^{0}=39.3 \mathrm{GPa}, \quad C_{22}^{0}=74.1 \mathrm{GPa}, \\
& e_{1}^{0}=0.347 \mathrm{C} / \mathrm{m}^{2}, \quad e_{2}^{0}=-0.16 \mathrm{C} / \mathrm{m}^{2},
\end{aligned}
$$




$$
\begin{aligned}
& \eta_{11}^{0}=9.03 \times 10^{-11} \mathrm{C}^{2} / \mathrm{Nm}^{2} \\
& P_{11}^{0}=-2.94 \times 10^{-6} \mathrm{C} / \mathrm{m}^{2} \mathrm{~K} \\
& \lambda_{r 0}=12.9 \mathrm{~W} / \mathrm{mK} .
\end{aligned}
$$

For convenience, some dimensionless values are defined as below:

$$
\left\{\begin{array}{l}
\bar{r}=\frac{r}{r_{b}}, \quad \bar{T}=\frac{T}{T_{0}}, \quad \bar{\sigma}_{i j}=\frac{\sigma_{i j}}{\alpha_{0} Y_{0} T_{0}}, \\
\bar{\varepsilon}_{i j}=\frac{\varepsilon_{i j}}{\alpha_{0} T_{0}}, \quad \bar{u}_{r}=\frac{u_{r}}{\alpha_{0} T_{0} r_{b}}, \quad \bar{C}_{i j}^{0}=\frac{C_{i j}^{0}}{Y_{0}}, \\
\bar{\alpha}_{i}^{0}=\frac{\alpha_{i}^{0}}{\alpha_{0}} \quad \bar{e}_{i}^{0}=\frac{e_{i}^{0}}{Y_{0}\left|d_{0}\right|}, \quad \bar{\eta}_{i j}^{0}=\frac{\eta_{i j}^{0}}{Y_{0}\left|d_{0}\right|^{2}}, \\
\bar{P}_{i j}^{0}=\frac{P_{i j}^{0}}{\alpha_{0} Y_{0}\left|d_{0}\right|}, \quad \bar{\phi}=\frac{\phi\left|d_{0}\right|}{\alpha_{0} T_{0} r_{b}}
\end{array}\right.
$$

where the typical parameters of material properties used to normalize the numerical results are considered:

$$
\alpha_{0}=\alpha_{\theta}^{0}, \quad Y_{0}=42.8 \mathrm{GPa}, \quad d_{0}=-3.92 \times 10^{-12} \mathrm{C} / \mathrm{N} .
$$

Here, two sets of thermo-electro-elastic governing equations of a hollow thick cylinder in the dimensionless form are presented [5]:

$$
\begin{aligned}
\bar{C}_{11}^{0} \bar{r}^{m} \frac{\partial^{2} \bar{u}_{r}}{\partial \bar{r}^{2}} & +(m+1) \bar{C}_{11}^{0} \bar{r}^{m-1} \frac{\partial \bar{u}_{r}}{\partial \bar{r}} \\
& +\bar{r}^{m-2}\left(m \bar{C}_{12}^{0}-\bar{C}_{22}^{0}\right) \bar{u}_{r}+\bar{e}_{1}^{0} \bar{r}^{m} \frac{\partial^{2} \bar{\phi}}{\partial \bar{r}^{2}} \\
& +\bar{r}^{m-1}\left[\bar{e}_{1}^{0}(1+m)-\bar{e}_{2}^{0}\right] \frac{\partial \bar{\phi}}{\partial \bar{r}} \\
& -\left[(1+m+n) \bar{\beta}_{1}^{0}-\bar{\beta}_{2}^{0}\right] \bar{r}^{m+n-1} \bar{T} \\
& -\bar{\beta}_{1}^{0} \bar{r}^{m+n} \frac{\partial \bar{T}}{\partial \bar{r}}=0 \\
\bar{e}_{1}^{0} \bar{r}^{m} \frac{\partial^{2} \bar{u}_{r}}{\partial \bar{r}^{2}}+ & {\left[(1+m) \bar{e}_{1}^{0}+\bar{e}_{2}^{0}\right] \bar{r}^{m-1} \frac{\partial \bar{u}_{r}}{\partial \bar{r}} } \\
+ & m \bar{e}_{2}^{0} \bar{r}^{m-2} \bar{u}_{r}-\bar{\eta}_{11}^{0} \bar{r}^{m} \frac{\partial^{2} \bar{\phi}}{\partial \bar{r}^{2}} \\
& (1+m) \bar{\eta}_{11}^{0} \bar{r}^{m-1} \frac{\partial \bar{\phi}}{\partial \bar{r}}(1+m+n) \\
\overline{\mathrm{P}}_{11}^{0} \bar{r}^{m+n-1} \bar{T}^{m}+\overline{\mathrm{P}}_{11}^{0} \bar{r}^{m+n} \frac{\partial \bar{T}}{\partial \bar{r}}=0 & \\
& (600
\end{aligned}
$$

where:

$$
\left\{\begin{array}{l}
\bar{\beta}_{1}^{0}=\bar{C}_{11}^{0} \bar{\alpha}_{2}^{0}+\bar{C}_{12}^{0} \bar{\alpha}_{3}^{0} \\
\bar{\beta}_{2}^{0}=\bar{C}_{21}^{0} \bar{\alpha}_{2}^{0}+\bar{C}_{22}^{0} \bar{\alpha}_{3}^{0}
\end{array}\right.
$$

In the sequel, the solution is assumed to be in the following form:

$$
\left\{\begin{array}{l}
\bar{u}_{r}=\bar{u}_{r c}+\bar{u}_{r p} \\
\bar{\phi}=\bar{\phi}_{c}+\bar{\phi}_{p}
\end{array}\right.
$$

The first term on the right-hand side of the above relation with subscript $c$ indicates the homogenous solution and the other one gives the particular solution. In the first step, the particular solution can be written as:

$$
\left\{\begin{array}{l}
\bar{u}_{r p}(\bar{r})=\mathrm{X}_{1} \bar{r}^{n-k+1}+\mathrm{X}_{2} \bar{r}^{n+1} \\
\bar{\phi}_{p}(\bar{r})=\mathrm{X}_{3} \bar{r}^{n-k+1}+\mathrm{X}_{4} \bar{r}^{n+1}
\end{array}\right.
$$

By substituting Eq. (63) into Eqs. (59) and (60), the unknown coefficients $\mathrm{X}_{i}(i=1,2,3,4)$ can be extracted. Utilizing the change of variable $\bar{r}=e^{s}$, the homogeneous expression of governing equations can be shown as $[5]$ :

$$
\begin{aligned}
& \left(\bar{D}^{2}+m \bar{D}-\alpha\right) \bar{u}_{r c}+\left[\bar{D}^{2}+(m-\beta) \bar{D}\right] \bar{\Phi}_{c}=0 \\
& {\left[\bar{D}^{2}+(m+\beta) \bar{D}+\beta m\right] \bar{u}_{r c}-\gamma\left(\bar{D}^{2}+m \bar{D}\right) \bar{\Phi}_{c}=0}
\end{aligned}
$$

where $\bar{D}=\mathrm{d} / \mathrm{d} s$,

$$
\begin{aligned}
& \alpha=\frac{\bar{C}_{22}^{0}-\bar{C}_{12}^{0}}{\bar{C}_{11}^{0}}, \quad \beta=\frac{\bar{e}_{2}^{0}}{\bar{e}_{1}^{0}}, \quad \gamma=\frac{\bar{C}_{11}^{0} \bar{\eta}_{11}^{0}}{\left(\bar{e}_{1}^{0}\right)^{2}}, \\
& \bar{\Phi}_{c}=\frac{\bar{e}_{1}^{0}}{\bar{C}_{11}^{0}} \bar{\phi}_{c} .
\end{aligned}
$$

From homogeneous Eqs. (64) and (65), the following equation can be obtained:

$$
\begin{aligned}
& \left\{(\gamma+1)\left(\bar{D}^{3}+2 m \bar{D}^{2}\right)\right. \\
& -\left[\beta^{2}+\alpha \gamma-m^{2}(\gamma+1)-m \beta\right] \\
& \left.\bar{D}-m\left(\beta^{2}-\beta m+\alpha \gamma\right)\right\} \bar{u}_{r c}=0 .
\end{aligned}
$$

Considering the general solution $\bar{u}_{r c}=e^{(\lambda s)}$ leads to the characteristic equation:

$$
\lambda^{3}+a_{1} \lambda^{2}+a_{2} \lambda+a_{3}=0
$$

where:

$$
\left\{\begin{array}{l}
a_{1}=2 m, a_{2}=-\frac{1}{1+\gamma}\left[\beta^{2}+\alpha \gamma-m^{2}(1+\gamma)-m \beta\right] \\
a_{3}=-\frac{m}{1+\gamma}\left(\beta^{2}-m \beta+\alpha \gamma\right)
\end{array}\right.
$$

According to the material properties selected in this example, we will obtain three distinct real roots and the general solutions of depending variables are as follows [5]: 


$$
\begin{aligned}
& \bar{u}_{r c}=\sum_{j=1}^{3} U_{j} \bar{r}^{\lambda_{j}}, \\
& \bar{\phi}_{c}=\sum_{j=1}^{3} M_{j} U_{j} \bar{r}^{\lambda_{j}}+M_{4} U_{4},
\end{aligned}
$$

where:

$$
\left\{\begin{array}{l}
M_{j}=\frac{\bar{C}_{11}^{0}}{\bar{e}_{1}^{0} \beta \gamma}\left[(\gamma+1) \lambda_{j}+m \gamma+m+\beta+\frac{m \beta-\alpha \gamma}{\lambda_{j}}\right] \\
j=1,2,3 \\
M_{4}=\frac{\bar{C}_{11}^{0}}{\bar{e}_{1}^{0}}
\end{array}\right.
$$

Assuming that the inner and outer surfaces of the cylinder are traction-free, the electro-elastic boundary conditions could be expressed as follows:

$$
\left\{\begin{array}{l}
\sigma_{r r}(\bar{r}=0.7)=0, \quad \sigma_{r r}(\bar{r}=1)=0 \\
\varphi(\bar{r}=0.7)=0, \quad \varphi(\bar{r}=1)=0
\end{array}\right.
$$

Moreover, the boundary conditions corresponding to the heat conduction equation are considered to be:

$$
\left\{\begin{array}{l}
T(\bar{r}=0.7)=0 \\
T(\bar{r}=1.0)=T_{0}
\end{array}\right.
$$

The inhomogeneous parameters $l=0.01$ and $b=0.01$ are considered for the numerical calculations. The variations of temperature, radial displacement and the electric potential of an FGP cylinder along the thickness direction are depicted in Figures 9-11 in terms of several inhomogeneous parameters $m$. It can be observed that the obtained results from the proposed combined FFT- $p$ FE method have a good agreement with the exact solution.

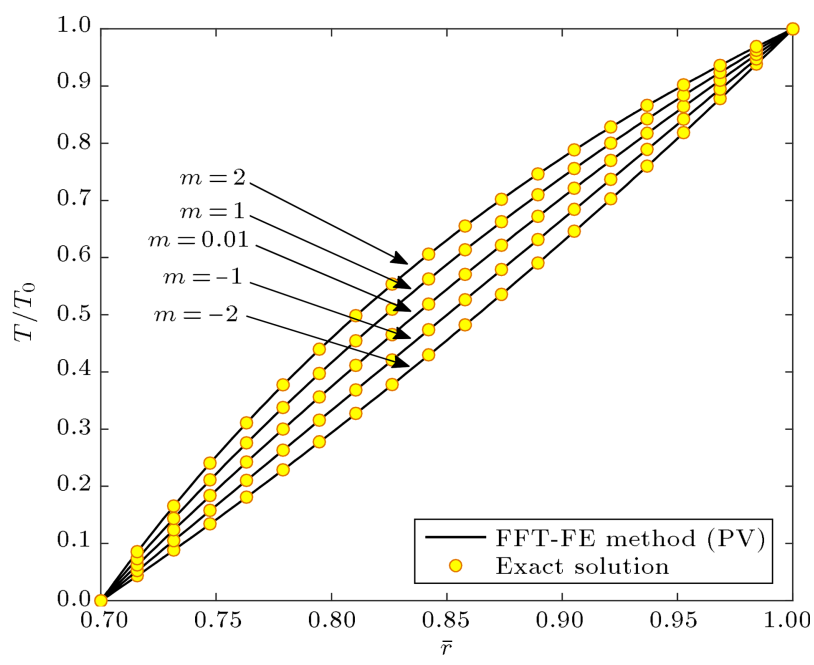

Figure 9. Temperature distribution along the thickness of cylinder $(p=7, \mathrm{Nel}=80)$.

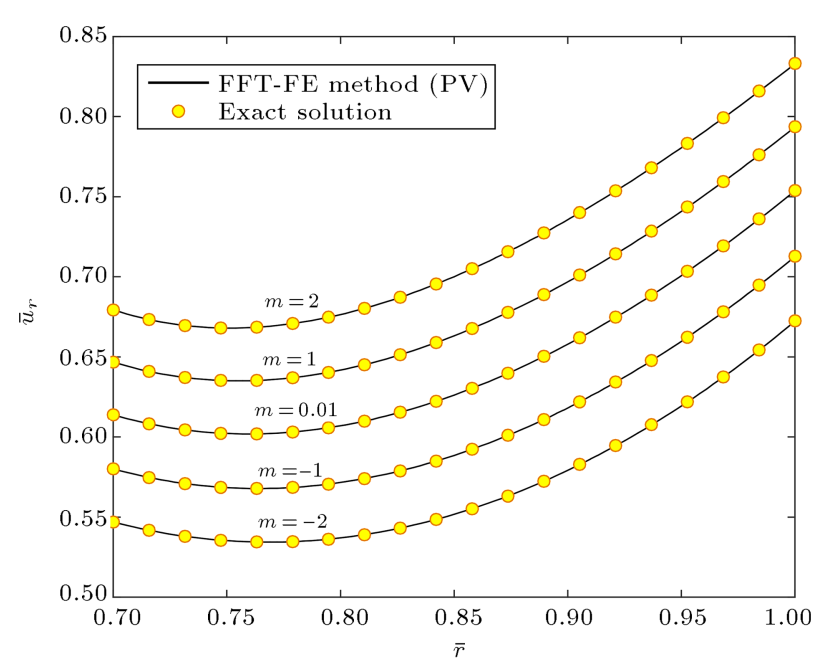

Figure 10. Distribution of radial displacement along the thickness of cylinder $(p=7, \mathrm{Nel}=80)$.

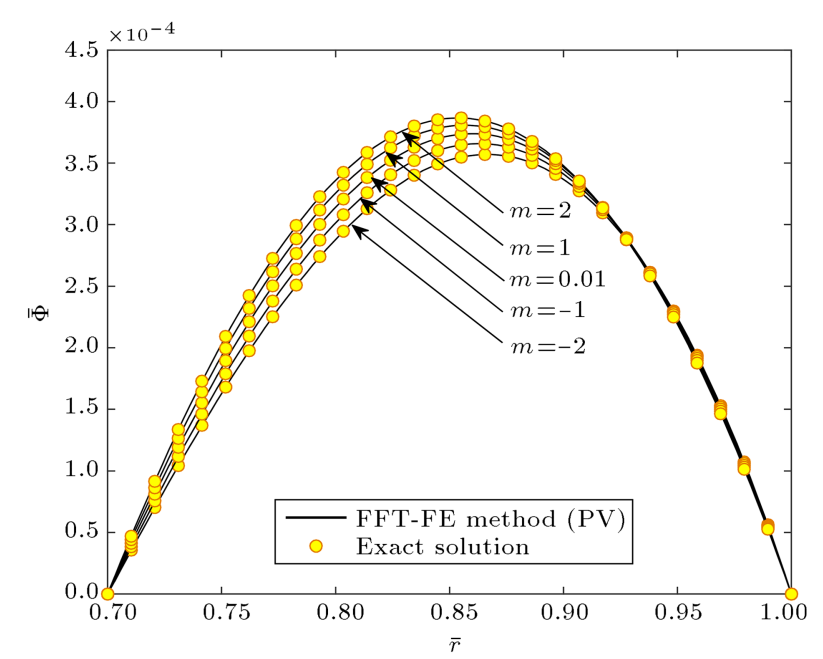

Figure 11. Distribution of electric potential along the thickness of cylinder $(p=7, \mathrm{Nel}=80)$.

As the verification process has been completed, the results of electro-thermo-elastic analysis of an FGP hollow cylinder under asymmetric thermal loadings are presented. The power index of material properties and the temperature boundary condition in the outer radii of the hollow cylinder are considered to be as follows:

$$
\begin{aligned}
& m=2, \quad d=0.01, \quad l=0.01, \\
& \bar{T}(\bar{r}=1, \theta)=\sin 2 \theta .
\end{aligned}
$$

Figure 12 shows the asymmetric two-dimensional distribution of the temperature field on the cylinder section. In this analysis, $N_{e l}=60$ hierarchic elements along the thickness direction and $N=80$ grid points along the circumferential direction are considered to obtain the results. Harmonic variation of the unknown variables, including the radial and circumferential displacements and electric potential are shown in Figures 13-15. Post-processing is then accomplished and 


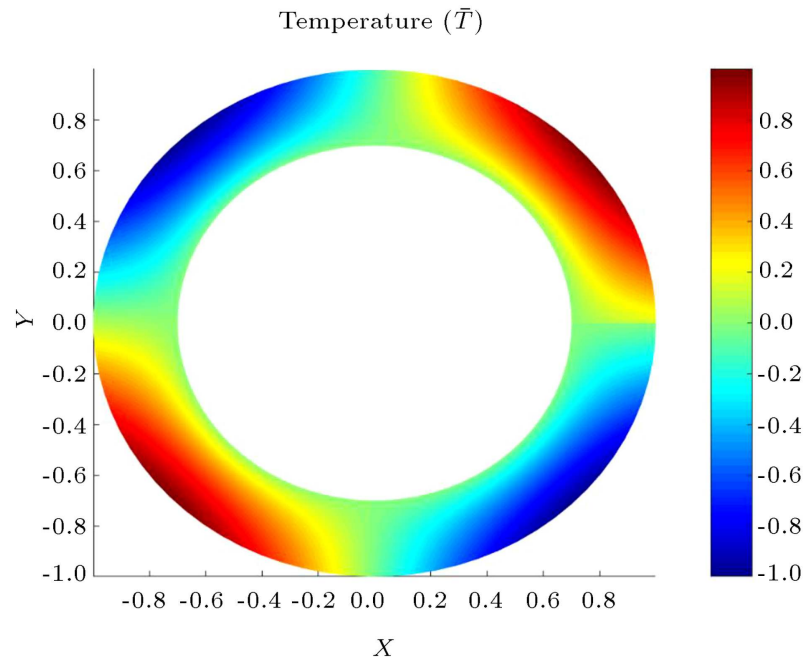

Figure 12. Two-dimensional asymmetric distribution of temperature.

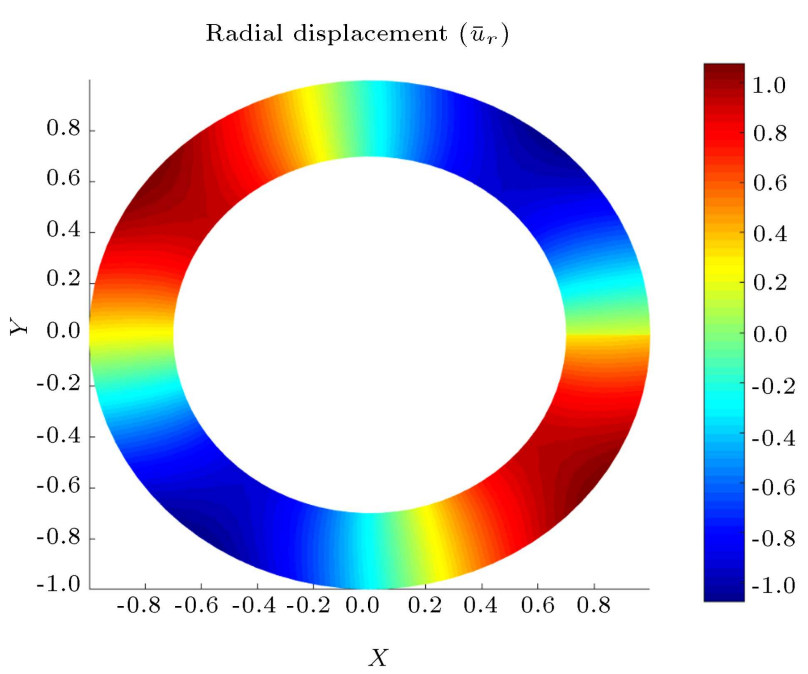

Figure 13. Two-dimensional asymmetric distribution of radial displacement.

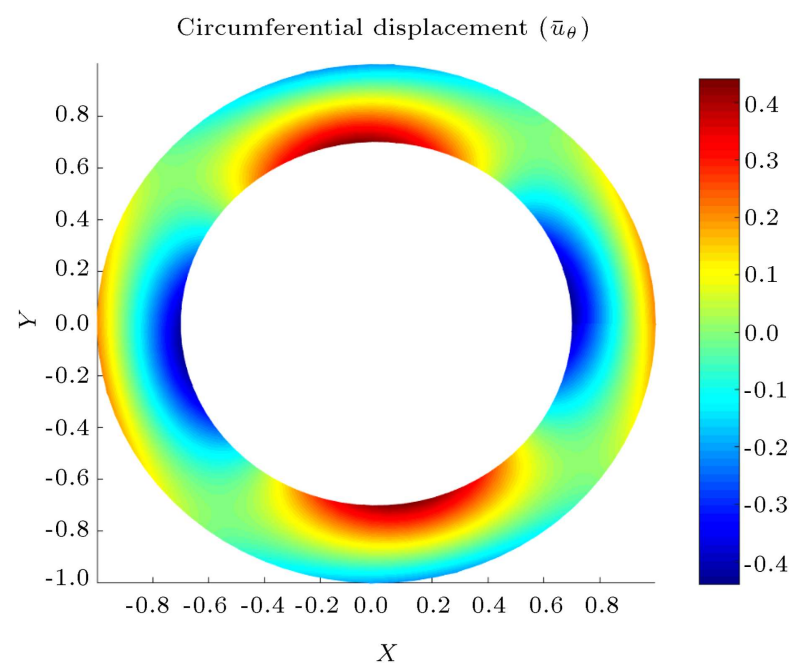

Figure 14. Two-dimensional asymmetric distribution of circumferential displacement.

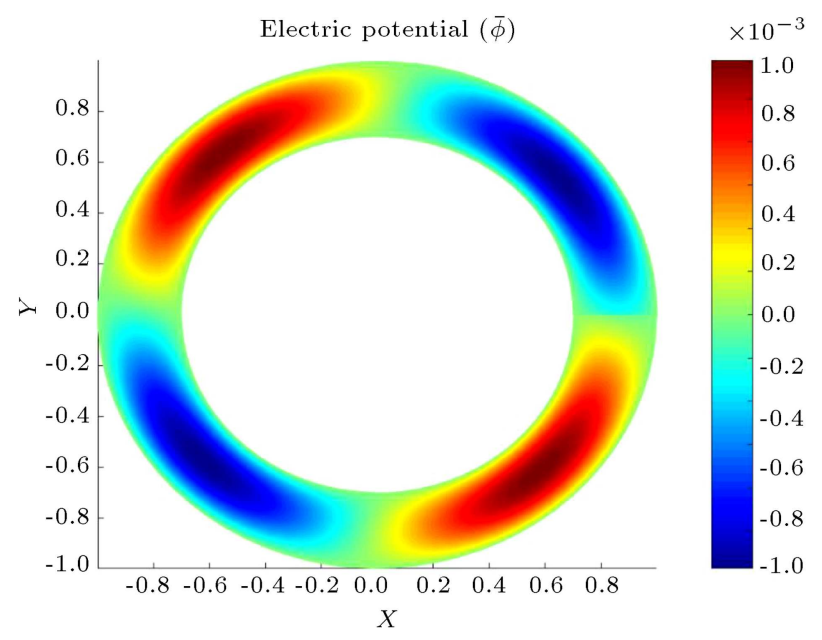

Figure 15. Two-dimensional asymmetric distribution of electric potential.

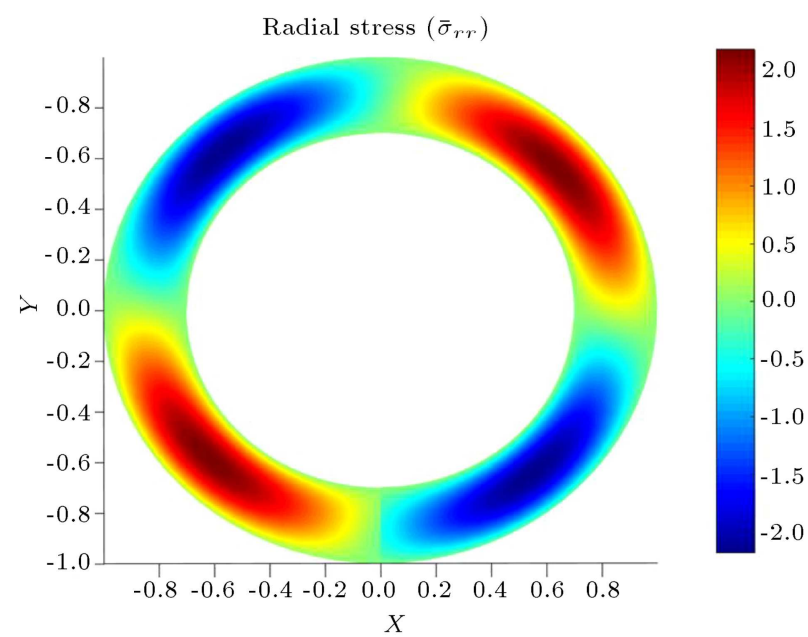

Figure 16. Two-dimensional asymmetric distribution of radial stresses.

the distribution of radial and hoop stresses are depicted in Figures 16 and 17.

\subsection{D FFT-pFE results (by quadratic Lagrange elements)}

4.2.1. Heat conduction of an FGP cylinder

As mentioned previously, the main idea of the present mixed method is to reduce the computational efforts by eliminating one of the spatial dimensions (i.e. circumferential). This feature is considered to be prominent in $3 \mathrm{D}$ analysis in which there exist a large number of computations. To verify the proposed mixed FFT- $p$ FE method for 3D thermal analysis, a thick hollow cylinder under thermal excitations is modeled in the ABAQUS software. Thermal boundary condition relevant to the inner radii of the hollow cylinder is $T(r=0.6, z, \theta)=$ $T_{0}\left(z^{2}\right) e^{\sin 2 \theta}$. The boundary temperature of the outer surface of the cylinder is considered to be unchanged at $T=0(r=1.0)$. Mutually, heat fluxes through the two ends of the cylinder $(z=0,4)$ are assumed to vanish. 


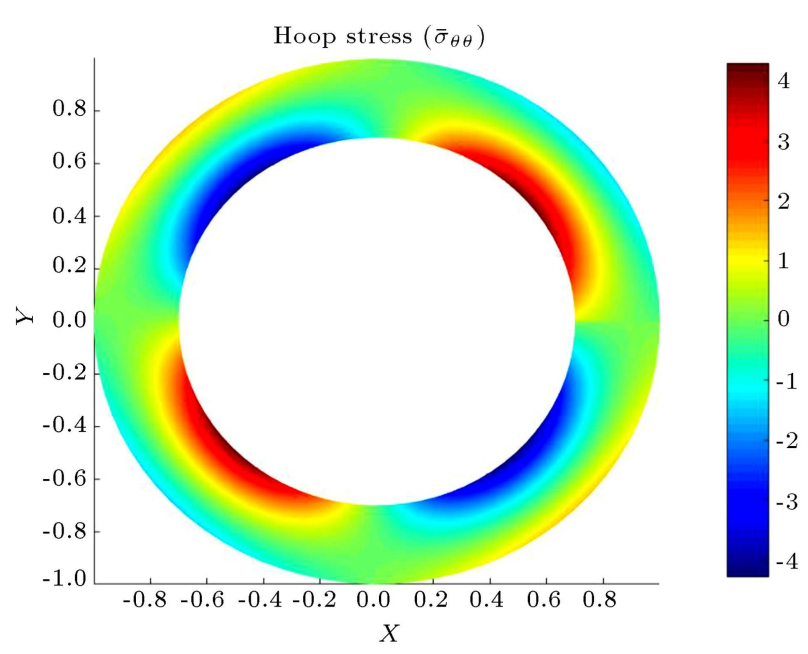

Figure 17. Two-dimensional asymmetric distribution of hoop stresses.

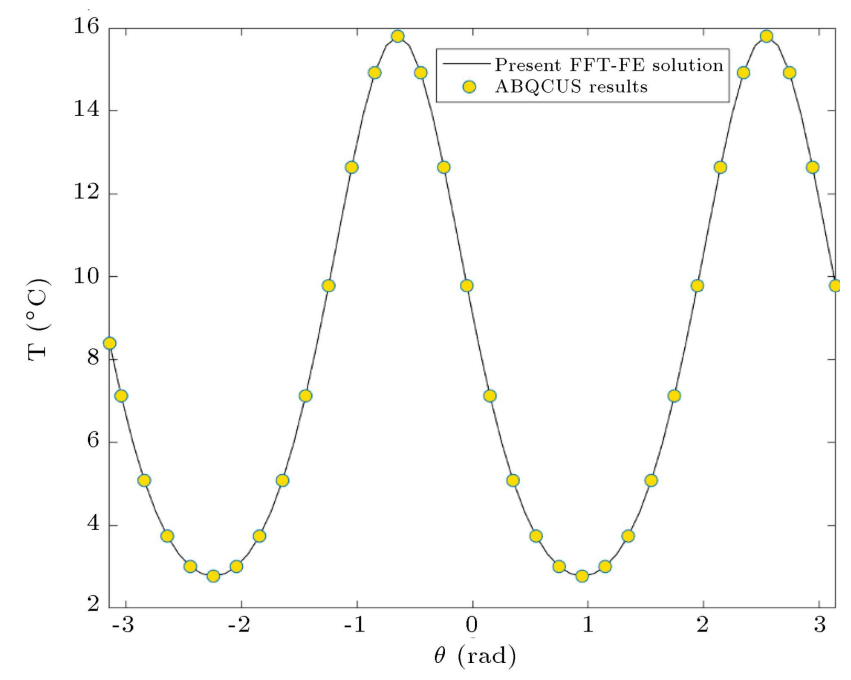

Figure 18. Distribution of the temperature field in the middle surface of the cylinder $(z=4, r=0.8)$.

For the case of simplicity, we used ABAQUS ability in modeling asymmetric loading employing Analytical Field subroutine. Considering the above boundary conditions, the temperature field variable can be extracted for all nodal points across the cylinder section. For better demonstration, paths including nodal points (e.g. $r=0.8$ path of the $z=4$ cross-section) are selected and the corresponding temperature nodal values are compared to those extracted from the FFT$p$ FE method (Figure 18).

\subsubsection{Thermo-electro-elastic responses}

After solving the heat conduction equation of an FGP hollow cylinder, the desired temperature field is used in the thermo-electro-elastic analysis. To obtain the results, the properties of the PZT-4 as a well-known piezoelectric material [50] are considered here. As an arbitrary boundary condition, in this study, we con-

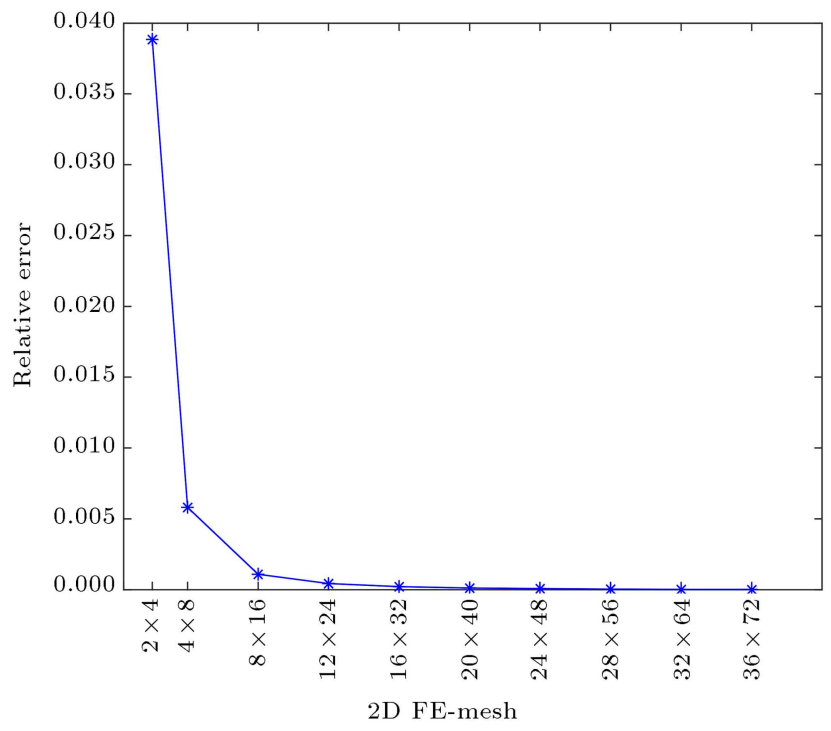

Figure 19. Convergence study of the FFT- $p$ FE method for thermo-electro-elastic analyzing.

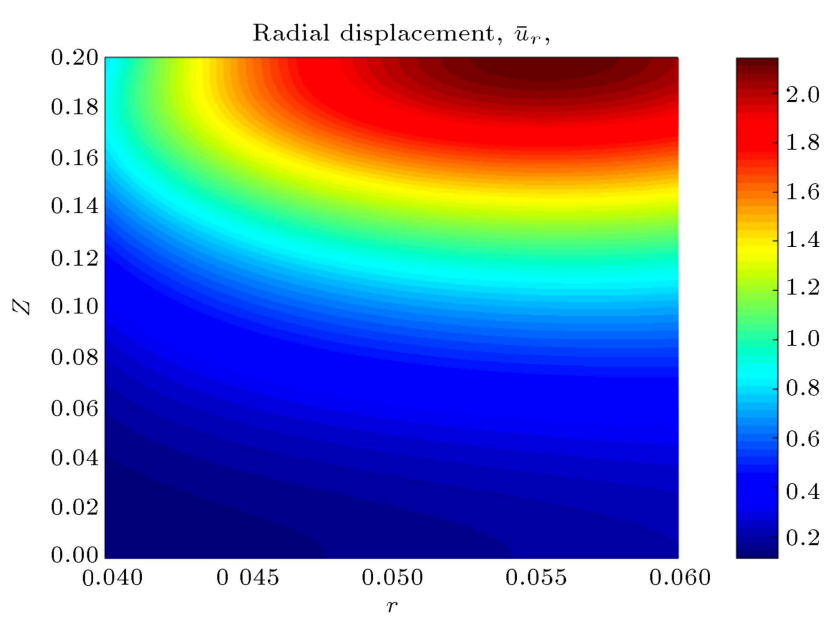

Figure 20. Contour plot of the radial component of displacement field across the $N=1$ surface.

strained the axial component of the displacement field at both ends of the FGP hollow cylinder. Electrical conditions at the inner and outer cylindrical surfaces are considered to be open circuits. So, we have:

$$
\sigma_{r r}=\sigma_{r z}=D_{r}=0, \quad \text { at } \quad r=a, b .
$$

To illustrate the convergence behavior and computational efficiency of the FFT- $p$ FE method in the 3D thermo-electro-elastic analysis, Figure 19 is presented. Radial displacement at the middle surface of the FGP hollow cylinder is considered to estimate the relative error. $n_{r} \times n_{z}$, labeled at the horizontal axis of Figure 19, indicates mesh density in the meridian section of the FGP cylinder. As shown in Figures 20 and 21, the contour plot of the non-dimensional components of the displacement field in the radial and circumferential directions are depicted. Consequently, 2D distribution 


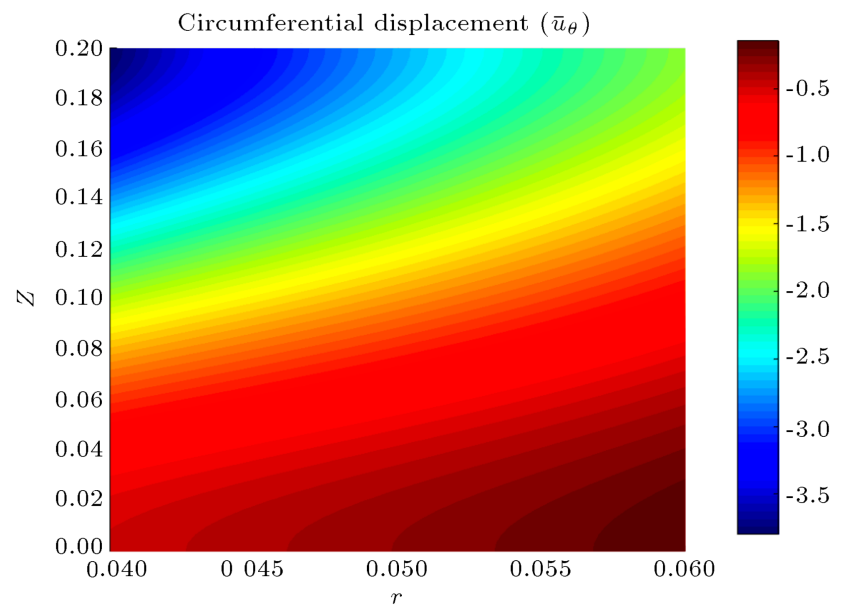

Figure 21. Contour plot of the circumferential component of displacement field across the $N=1$ surface.

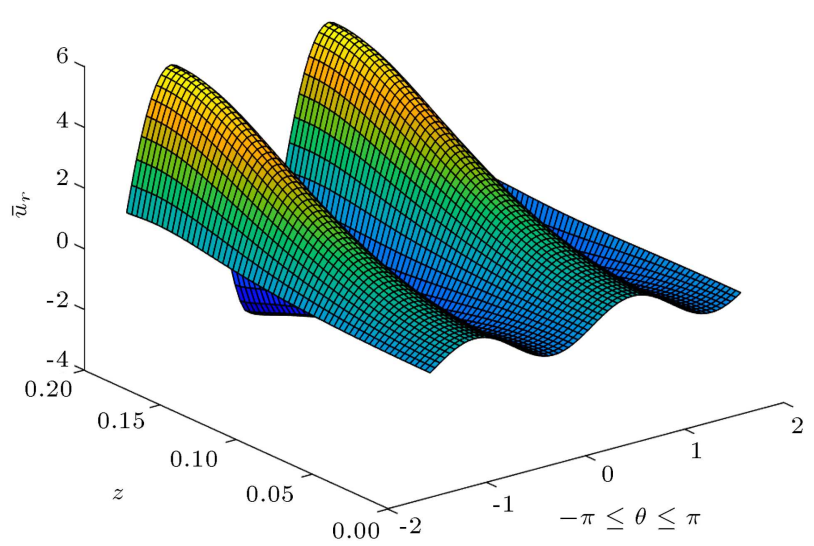

Figure 22. 2D distribution of the radial displacement across the middle surface of the Functionally Graded Piezoelectric (FGP) cylinder.

of the displacement components and electric potential of the FGP cylinder are shown in Figures 22-25.

\section{Conclusions}

A combination of the Fast Fourier Transform (FFT) technique and $p$-version of the Finite Element Method (FEM) was performed in this paper for the electrothermo-elastic analysis of a hollow Functionally Graded Piezoelectric (FGP) cylinder. By considering the geometric feature of the shells of revolution, the integral transform technique is appropriately used in the circumferential direction. Therefore, the governing equations can be divided into a set of harmonics. Afterwards, pertinent individual equations are discretized using higher-order FEM. This procedure decreases one dimension of the related governing equations. Thus, a significant reduction in computational cost can be achieved. In this regard, the effects of the FFT grid-size and the higher-order interpolation functions are indicated. As expected, as the FFT grid size

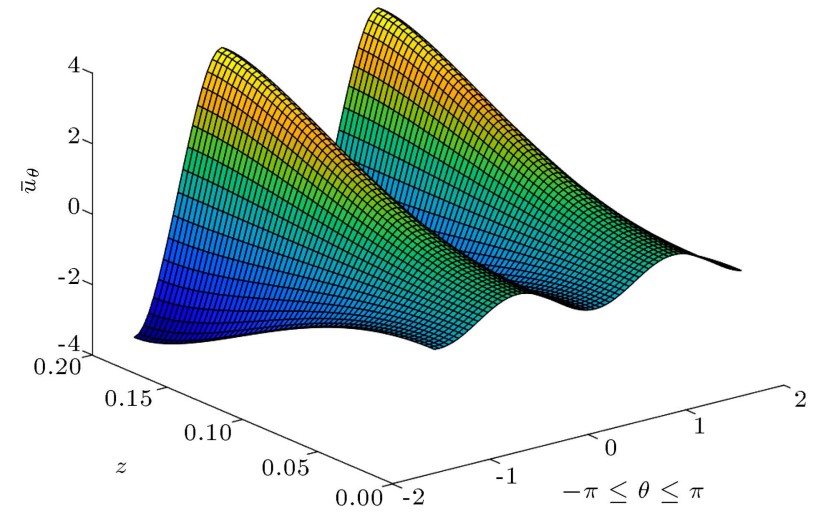

Figure 23. 2D distribution of the circumferential displacement across the middle surface of the Functionally Graded Piezoelectric (FGP) cylinder.

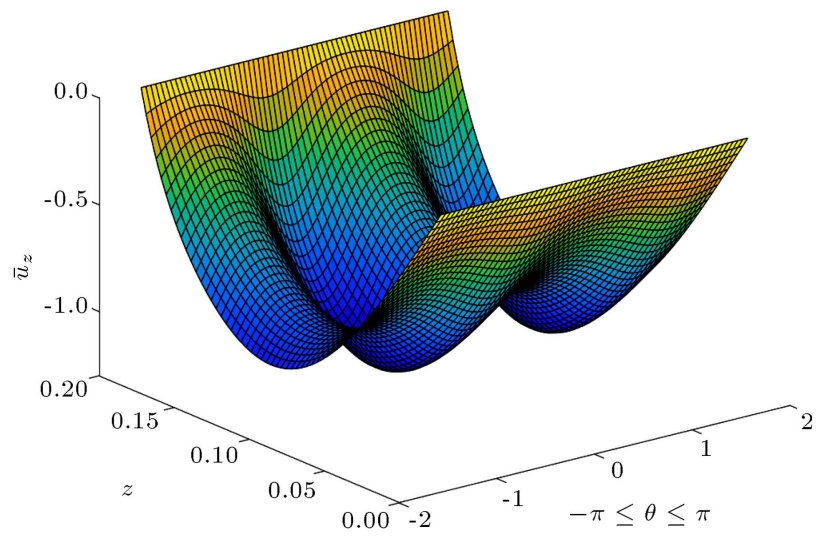

Figure 24. 2D distribution of the axial displacement across the middle surface of the Functionally Graded Piezoelectric (FGP) cylinder.

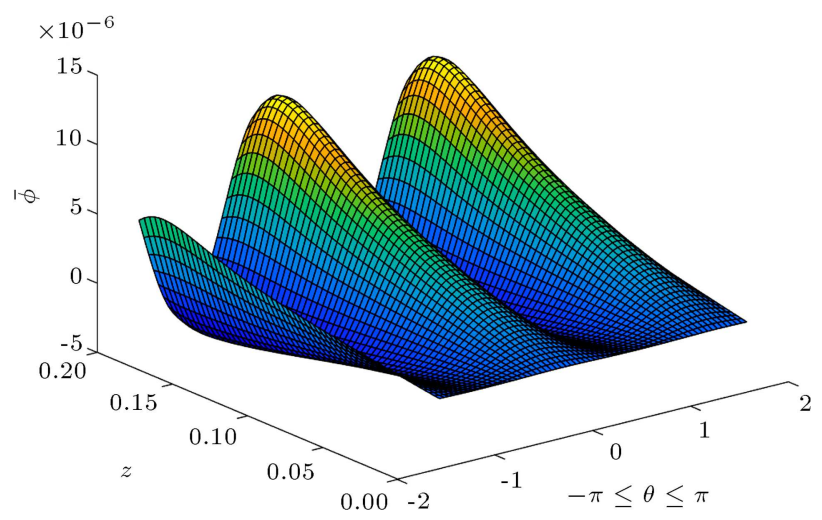

Figure 25. 2D distribution of the electric potential across the middle surface of the FGP cylinder.

increases, the trigonometric Fourier functions show the highest convergence rate. It can be concluded from the convergence study that the FFT technique has an exponentially varying convergence behavior whereas the hierarchic FEM shows an algebraic behavior. Nevertheless, it is apparent from the results that the rate of convergence of the hierarchic elements is much higher 
than that of the linear finite elements. Since the FFT technique needs to be implemented on the strong form and the FEM deals with the weak formulation, Hamilton's principle can be used to gather both the strong and weak form of the governing equations, simultaneously. The FFT technique helps us to employ asymmetric thermal loadings. The results showed that the proposed method is in good agreement with the exact analytical solutions from the literature. The proposed FFT- $p$ FE method not only has the ability of FEM in modeling complex geometric modeling but also has the simplicity and accuracy of FFT. As a future extension of this work, the proposed method can be extended to the three-dimensional electro-thermoelastic analysis of homogeneous, composite, and FG thick shells of revolution.

\section{Nomenclature}

$C_{i j} \quad$ Material properties coefficients

$D_{i} \quad$ Electric displacements

$e_{i j} \quad$ Piezoelectric constants

$E_{i} \quad$ Components of electric field

$\hat{K}_{i j} \quad$ Components of stiffness matrix

$N_{p}(\xi) \quad$ Legendre polynomial of degree $p$

$\hat{Q}_{i} \quad$ Components of temperature gradient vector

$\hat{T}^{k} \quad$ Temperature variable of $k$ th harmonic in Fourier space

$\left(\hat{u}_{i}\right)_{j}^{k} \quad$ Nodal values of displacement components in $k$ th harmonic

$w \quad$ Vector of weighting functions

$\alpha_{i} \quad$ Coefficients of thermal expansion

$\lambda_{r} \quad$ Thermal conductivity coefficient

$\psi_{j} \quad$ Basis functions

$\eta_{i j} \quad$ Dielectric constants

$\sigma_{i j} \quad$ Components of stress tensor

$\varepsilon_{i j} \quad$ Components of strain tensor

$P_{i} \quad$ Pyroelectric constants

\section{References}

1. Moosaie, A. "Axisymmetric steady temperature field in FGM cylindrical shells with temperature-dependent heat conductivity and arbitrary linear boundary conditions", Archives of Mechanics, 67(3), pp. 233-251 (2015).

2. Jabbari, M., Nejad, M.Z., and Ghannad, M. "Thermoelastic analysis of axially functionally graded rotating thick cylindrical pressure vessels with variable thickness under mechanical loading", International Journal of Engineering Science, 96, pp. 1-18 (2015).
3. Moosaie, A. and Panahi-Kalus, H. "Thermal stresses in an incompressible FGM spherical shell with temperature-dependent material properties", ThinWalled Structures, 120, pp. 215-224 (2017).

4. Takagi, K., Li, J.-F., Yokoyama, S., et al. "Design and fabrication of functionally graded PZT/Pt piezoelectric bimorph actuator", Science and Technology of Advanced Materials, 3(2), pp. 217-224 (2002).

5. Ootao, Y., Akai, T., and Tanigawa, Y. "Transient piezothermoelastic analysis for a functionally graded thermopiezoelectric hollow cylinder", Journal of Thermal Stresses, 31(10), pp. 935-955 (2008).

6. Wu, C.-P. and Syu, Y.-S. "Exact solutions of functionally graded piezoelectric shells under cylindrical bending", International Journal of Solids and Structures, 44(20), pp. 6450-6472 (2007).

7. Alashti, R.A., Khorsand, M., and Tarahhomi, M. "Thermo-elastic analysis of a functionally graded spherical shell with piezoelectric layers by differential quadrature method", Scientia Iranica, 20(1), pp. 109119 (2013).

8. Dehghan, M., Nejad, M.Z., and Moosaie, A. "Thermoelectro-elastic analysis of functionally graded piezoelectric shells of revolution: Governing equations and solutions for some simple cases", International Journal of Engineering Science, 104, pp. 34-61 (2016).

9. Nejad, M.Z., Jabbari, M., and Ghannad, M. "A general disk form formulation for thermo-elastic analysis of functionally graded thick shells of revolution with arbitrary curvature and variable thickness", Acta Mechanica, 228(1), pp. 215-231 (2017).

10. Santos, H., Soares, C.M.M., Soares, C.A.M., et al. "A semi-analytical finite element model for the analysis of laminated 3D axisymmetric shells: bending, free vibration and buckling", Composite Structures, 71(3), pp. 273-281 (2005).

11. Santos, H., Soares, C.M.M., Soares, C.A.M., et al. "A finite element model for the analysis of 3D axisymmetric laminated shells with piezoelectric sensors and actuators", Composite Structures, 75(1), pp. 170-178 (2006).

12. Santos, H., Soares, C.M.M., Soares, C.A.M., et al. "A semi-analytical finite element model for the analysis of cylindrical shells made of functionally graded materials under thermal shock", Composite Structures, 86(1), pp. 10-21(2008).

13. Santos, H., Soares, C.M.M., Soares, C.A.M., et al. "A finite element model for the analysis of 3D axisymmetric laminated shells with piezoelectric sensors and actuators: bending and free vibrations", Computers \& Structures, 86(9), pp. 940-947 (2008).

14. Santos, H., Soares, C.M.M., Soares, C.A.M., et al. "A semi-analytical finite element model for the analysis of cylindrical shells made of functionally graded materials", Composite Structures, 91(4), pp. 427-432 (2009). 
15. Noor, A.K. and Peters, J.M. "Stress and vibration analyses of anisotropic shells of revolution", International Journal for Numerical Methods in Engineering, 26(5), pp. 1145-1167 (1988).

16. Sheinman, I. and Weissman, S. "Coupling between symmetric and antisymmetric modes in shells of revolution", Journal of Composite Materials, 21(11), pp. 988-1007 (1987).

17. Sivadas, K. and Ganesan, N. "Effect of coupling between symmetric and antisymmetric modes in composite thick shells of revolution", Finite Elements in Analysis and Design, 11(1), pp. 9-18 (1992).

18. Loghman, A., Nasr, M., and Arefi, M. "Nonsymmetric thermomechanical analysis of a functionally graded cylinder subjected to mechanical, thermal, and magnetic loads", Journal of Thermal Stresses, 40(6), pp. $1-18$ (2017).

19. Chen, W., Bian, Z., Lv, C., et al. "3D free vibration analysis of a functionally graded piezoelectric hollow cylinder filled with compressible fluid", International Journal of Solids and Structures, 41(3-4), pp. 947-964 (2004).

20. Liang, X., Deng, Y., Cao, Z., et al. "Three-dimensional dynamics of functionally graded piezoelectric cylindrical panels by a semi-analytical approach", Composite Structures, 226, p. 111176 (2019).

21. Gao, W., Qin, Z., and Chu, F. "Wave propagation in functionally graded porous plates reinforced with graphene platelets", Aerospace Science and Technology, 102, p. 105860 (2020).

22. Canuto, C.G., Hussaini, M.Y., Quarteroni, A., et al., Spectral Methods: Fundamentals in Single Domains, springer (2010).

23. Dehghan, M. and Baradaran, G.H. "Buckling and free vibration analysis of thick rectangular plates resting on elastic foundation using mixed finite element and differential quadrature method", Applied Mathematics and Computation, 218(6), pp. 2772-2784 (2011).

24. Malekzadeh, P. and Karami, G. "A mixed differential quadrature and finite element free vibration and buckling analysis of thick beams on two-parameter elastic foundations", Applied Mathematical Modelling, 32(7), pp. 1381-1394 (2008).

25. Lin, J.-Y. and Chen, H.-T. "Radial axisymmetric transient heat conduction in composite hollow cylinders with variable thermal conductivity", Engineering Analysis with Boundary Elements, 10(1), pp. 27-33 (1992).

26. Liu, T.-W., Xu, H.-H., and Qiu, X.-L. "A combination method of mixed multiscale finite-element and laplace transform for flow in a dual-permeability system", International Scholarly Research Notices, 2012, p. 202893 (2012).

27. Entezari, A., Filippi, M., Carrera, E., et al. "3D-wave propagation in generalized thermoelastic functionally graded disks", Composite Structures, 206, pp. 941-951 (2018).
28. Heydarpour, Y., Malekzadeh, P., Dimitri, R., et al. "Thermoelastic analysis of functionally graded cylindrical panels with piezoelectric layers", Applied Sciences, 10(4), p. 1397 (2020).

29. Qin, Z., Pang, X., Safaei, B., et al. "Free vibration analysis of rotating functionally graded CNT reinforced composite cylindrical shells with arbitrary boundary conditions", Composite Structures, 220, pp. 847-860 (2019).

30. Qin, Z., Yang, Z., Zu, J., et al. "Free vibration analysis of rotating cylindrical shells coupled with moderately thick annular plates", International Journal of Mechanical Sciences, 142, pp. 127-139 (2018).

31. Qin, Z., Zhao, S., Pang, X., et al. "A unified solution for vibration analysis of laminated functionally graded shallow shells reinforced by graphene with general boundary conditions", International Journal of $\mathrm{Me}$ chanical Sciences, 170, p. 105341 (2020).

32. Safaei, B. "The effect of embedding a porous core on the free vibration behavior of laminated composite plates", Steel Compos. Struct, 35(5), pp. 659-670 (2020).

33. Safaei, B., Ahmed, N., and Fattahi, A. "Free vibration analysis of polyethylene/CNT plates", The European Physical Journal Plus, 134(6), p. 271 (2019).

34. Cho, C. and Ahn, S. "Transient thermoelastic analysis of disk brake using the fast Fourier transform and finite element method", Journal of Thermal Stresses, 25(3), pp. 215-243 (2002).

35. Floquet, A. and Dubourg, M. "Nonaxisymmetric effects for three-dimensional analysis of a brake", Journal of Tribology, 116(3), pp. 401-407 (1994).

36. Floquet, A. and Dubourg, M.-C. "Realistic braking operation simulation of ventilated disk brakes", Journal of Tribology, 118(3), pp. 466-472 (1996).

37. Jin, G., Ma, X., Shi, S., et al. "A modified Fourier series solution for vibration analysis of truncated conical shells with general boundary conditions", Applied Acoustics, 85, pp. 82-96 (2014).

38. Mohazzab, A.H. and Dozio, L. "Prediction of natural frequencies of laminated curved panels using refined 2D theories in the spectral collocation method", Curved Layer Struct, 2, pp. 1-14 (2015).

39. Mohazzab, A.H. and Dozio, L. "A spectral collocation solution for in-plane eigenvalue analysis of skew plates", International Journal of Mechanical Sciences, 94, pp. 199-210 (2015).

40. Xie, X., Zheng, H., and Jin, G. "Integrated orthogonal polynomials based spectral collocation method for vibration analysis of coupled laminated shell structures", International Journal of Mechanical Sciences, 98, pp. 132-143 (2015).

41. Szabó, B. and Babuska, I., Introduction to Finite Element Analysis, John Wiley and Sons (2011).

42. Adjerid, S., Aiffa, M., and Flaherty, J. "Hierarchical 
finite element bases for triangular and tetrahedral elements", Computer Methods in Applied Mechanics and Engineering, 190(22), pp. 2925-2941 (2001).

43. Yu, Z., Guo, X., and Chu, F. "A multivariable hierarchical finite element for static and vibration analysis of beams", Finite Elements in Analysis and Design, 46(8), pp. 625-631 (2010).

44. Tai, C.-Y. and Chan, Y. "A hierarchic high-order Timoshenko beam finite element", Computers \& Structures, 165, pp. 48-58 (2016).

45. Wang, H. and Qin, Q.H. "FE approach with Green's function as internal trial function for simulating bioheat transfer in the human eye", Archives of Mechanics, 62(6), pp. 493-510 (2010).

46. Cao, C., Qin, Q.-H., and Yu, A. "A new hybrid finite element approach for three-dimensional elastic problems", Archives of Mechanics, 64(3), pp. 261-292 (2012).

47. Zienkiewicz, O.C. and Taylor, R.L., The Finite Element Method: Solid Mechanics, Butterworthheinemann, (2000).

48. Duda, P. "Finite element method formulation in polar coordinates for transient heat conduction problems", Journal of Thermal Science, 25(2), pp. 188-194 (2016).

49. Jabbari, M., Sohrabpour, S., and Eslami, M. "Mechanical and thermal stresses in a functionally graded hollow cylinder due to radially symmetric loads", International Journal of Pressure Vessels and Piping, 79(7), pp. 493-449 (2002).

50. Alashti, R.A. and Khorsand, M. "Three-dimensional thermo-elastic analysis of a functionally graded cylindrical shell with piezoelectric layers by differential quadrature method", International Journal of Pressure Vessels and Piping, 88(5-7), pp. 167-180 (2011).

\section{Biographies}

Mehdi Dehghan received his MSc degree in Mechanical Engineering from the Shahid Bahonar University of Kerman in 2011, Iran and, his PhD degree in Mechanical Engineering from Yasouj University, Iran in 2018. He is interested in advanced numerical methods for the nonlinear analysis of three-dimensional shells of revolution in curvilinear coordinate systems.

Amin Moosaie received his BSc in Mechanical Engineering from Iran University of Science and Technology (IUST), Tehran, Iran, in 2006. He earned his MSc in Computational Mechanics and his $\mathrm{PhD}$ in Fluid Dynamics and Aerodynamics from Technische Universität München (TUM), Germany, in 2008 and 2011, respectively. He then started post-doctoral studies at TUM in the same subject, during which he took a sabbatical leave to the Polymer Physics Department at the ETH-Zurich, Switzerland, for three months in the Summer of 2011. He joined the Mechanical Engineering Department of Yasouj University, Iran, as a faculty member in 2012, where he is currently an Associate Professor. He is the Khwarizmi Award laureate of 2006 and has supervised the best MSc thesis of 2015 as elected by the Iranian Society of Mechanical Engineers (ISME). His primary research interests are computational mechanics, continuum mechanics, turbulence simulation (DNS/LES), drag reduction, and polymer dynamics in fluid flows.

Mohammad Zamani Nejad is an Associate Professor in the Department of Mechanical Engineering, College of Engineering, at Yasouj University, Iran. He received his $\mathrm{PhD}$ in College of Engineering from Tarbiat Modares University, Tehran, Iran. Moreover, He received his $\mathrm{BSc}$ and $\mathrm{MSc}$ degrees in Mechanical Engineering from Shiraz University and Mazandaran University, respectively. His research interest areas include thermo-elasto-plastic analysis and creep analysis of beams, plates, and shells. He has published several papers in international journals and serves as an editorial board member for some international journals. 